\title{
Type-Level Programming with Match Types
}

\author{
OLIVIER BLANVILLAIN, EPFL, Switzerland \\ JONATHAN IMMANUEL BRACHTHÄUSER, University of Tübingen, Germany \\ MAXIME KJAER, EPFL, Switzerland \\ MARTIN ODERSKY, EPFL, Switzerland
}

\begin{abstract}
Type-level programming is becoming more and more popular in the realm of functional programming. However, the combination of type-level programming and subtyping remains largely unexplored in practical programming languages. This paper presents match types, a type-level equivalent of pattern matching. Match types integrate seamlessly into programming languages with subtyping and, despite their simplicity, offer significant additional expressiveness. We formalize the feature of match types in a calculus based on System $\mathrm{F}_{<:}$ and prove its soundness. We practically evaluate our system by implementing match types in the Scala 3 reference compiler, thus making type-level programming readily available to a broad audience of programmers.

CCS Concepts: • Software and its engineering $\rightarrow$ Formal language definitions; Language features; Multiparadigm languages; Compilers; • Theory of computation $\rightarrow$ Type structures.
\end{abstract}

Additional Key Words and Phrases: Match types, Scala

\section{ACM Reference Format:}

Olivier Blanvillain, Jonathan Immanuel Brachthäuser, Maxime Kjaer, and Martin Odersky. 2022. Type-Level Programming with Match Types. Proc. ACM Program. Lang. 6, POPL, Article 37 (January 2022), 24 pages. https://doi.org/10.1145/3498698

\section{INTRODUCTION}

There is a growing interest in using type-level computation to increase the expressivity of type systems, express additional constraints on the type level, and thereby improve the safety of generalpurpose software. What used to be an exclusive feature of dependently typed languages is slowly becoming accessible to everyday programmers. GHC Haskell has been at the forefront of making this a reality and already provides several extensions to support type-level programming. While Haskell is certainly not the only language moving towards dependent types, the trend seems to be limited to pure functional programming languages.

We believe that type-level programming is not necessarily incompatible with other programming paradigms and that the current division exists mainly due to a lack of attention from the research community. Unfortunately, most of the existing research conducted in this domain is not directly applicable to languages with subtyping. Although the combination of subtyping and type-level programming has been studied extensively on the theoretical side, through the means of dependently typed systems [Aspinall 1995; Courant 2003; Hutchins 2010; Stone and Harper 2000; Yang and Oliveira 2017; Zwanenburg 1999], the practical side remains largely unexplored.

Authors' addresses: Olivier Blanvillain, EPFL, Switzerland, olivier.blanvillain@epfl.ch; Jonathan Immanuel Brachthäuser, University of Tübingen, Germany, jonathan.brachthaeuser@uni-tuebingen.de; Maxime Kjaer, EPFL, Switzerland, maxime. kjaer@alumni.epfl.ch; Martin Odersky, EPFL, Switzerland, martin.odersky@epfl.ch.

This work is licensed under a Creative Commons Attribution 4.0 International License.

(c) 2022 Copyright held by the owner/author(s).

2475-1421/2022/1-ART37

https://doi.org/10.1145/3498698

Proc. ACM Program. Lang., Vol. 6, No. POPL, Article 37. Publication date: January 2022. 
One notable exception is the TypeScript language, which recently introduced a new feature called conditional type, a type-level ternary operator based on subtyping. A conditional type, written $S$ extends $T$ ? Tt : Tf, reduces to Tt when $S$ is a subtype of $T$, to Tf when $S$ is not a subtype of $\mathrm{T}$, and is left unreduced when types variables do not allow to draw a conclusion. Unfortunately, the algorithm used to reduce such conditional types is both unsound and incomplete. Despite the unsoundness (discussed in Subsection 6.5), the addition of conditional types to TypeScript illustrates the practical need and timeliness of this feature.

This paper presents an alternative construct for type-level programming based on subtyping, which we call match types. As the name suggests, match types allow programmers to express types that perform pattern matching on types:

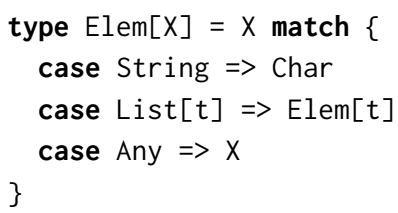

The example, which we explain in detail in Section 2, defines the type Elem by matching on the type parameter $\mathrm{X}$. We have implemented match types in the latest version of Scala, an industry-grade, production-ready compiler. Match types have received a great interest from the Scala community, and are already in active use.

In this paper, we explore the theoretical foundations of match types through the lens of a type system which extends System $\mathrm{F}_{<:}$with pattern matching at the term and type level. Our formalization serves two purposes: first, it gives a clear view on how we integrated match types in Scala's type system and precisely describes the changes needed on the subtyping relation to make this integration possible. Second, thanks to a type safety proof based on the standard progress and preservation theorems, it gives confidence that the design of match types is sensible and our implementation is sound.

Conditional types provide concrete evidence that our results are valuable beyond the context of Scala. Our results are directly applicable to TypeScript's type system and provide a clear path to fixing the unsoundness introduced by conditional types. Furthermore, we hope that match types can be useful as a reference for future designs of type-level programming features for languages with subtyping.

In summary, this paper makes the following contributions:

- We introduce programming with match types in Scala by means of an example and highlight the interaction of type-level programming and subtyping (Section 2).

- We formalize match types in the self-contained calculus System FM and prove it sound, providing a theoretical basis of our implementation (Section 3). The paper is accompanied by a mechanization of System FM, including proofs of progress and preservation.

- We describe our implementation of match types in the Scala compiler, discuss challenges, and relate the implementation to our formalization (Section 4).

- We evaluate match types in a case study, presenting a type-safe version of the NumPy library (Section 5).

- We motivate the design of our formalization relative to prior work, we review the extensive related work on type families in Haskell, and discuss the unsoundness of conditional types in TypeScript (Section 6). 


\section{OVERVIEW}

In this section, we offer a brief introduction of match types in Scala by inspecting the example from the previous section in more detail:

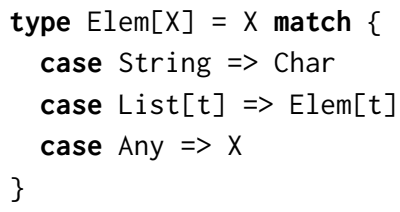

This example defines a type Elem parametrized by one type parameter $\mathrm{x}$. The right-hand side is defined in terms of a match on the type parameter - a match type. A match type reduces to one of its right-hand sides, depending on the type of its scrutinee. For example, the above type reduces as follows:

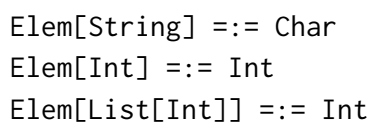

Here we use $S=:=T$ to denote type equality between the two types $S$ and $T$, witnessed by mutual subtyping. To reduce a match type, the scrutinee is compared to each pattern, one after the other, using subtyping. For example, although String is a subtype of both String and Any (the top of Scala's subtyping lattice), Elem[String] reduces to Char because the corresponding case appears first.

When the scrutinee type is a List, the match type Elem is defined recursively on the element type of the list. Hence, in our example Elem[List[Int]] first reduces to the type Elem[Int], and eventually to the type Int.

\subsection{A Lightweight Form of Dependent Typing}

Match types enable a lightweight form of dependent typing, since a term-level pattern matching expression can be typed accordingly at the type level as a match type. Consider the following function definition:

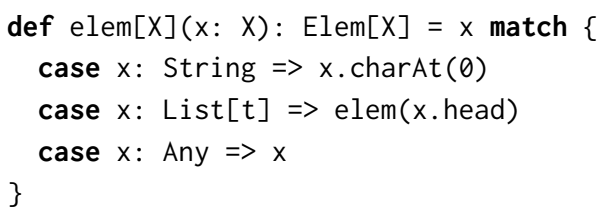

This definition is well-typed because the match expression in elem's body has the exact same scrutinee and pattern types as Elem[X] (the function's return type).

Thanks to Scala's type inference, a call to the elem function can have a result type that depends on a term-level parameter. For instance, in the expression elem(1), the Scala compiler infers the singleton type $X=1$ for elem's type parameter. This expression thus has type Elem[1], which reduces to Int (via Elem's third case). Similarly, in elem(x), the compiler infers the singleton type $x=x$.type and the expression has type Elem[x.type], which might reduce further at the callsite depending on $x$ 's type.

In both examples, singleton types create a dependency between a type parameter and a term, which, by transitivity, results in a lightweight form of dependent typing, that is, a dependency between a term parameter and a function's result type. 


\subsection{Disjointness}

Our design of match types induces an additional constraint on match type reduction: the scrutinee type must be known to be disjoint with all type patterns preceding the matching case. Informally, disjointness means that two types have no shared inhabitants.

The necessity for disjointness is best illustrated with an example. Consider Seq[Int], the type of integer sequences. Elem[Seq[Int]] does not reduce:

(1) Elem's first case does not apply because Seq[Int] is not a subtype of String.

(2) Elem's second case does not apply because Seq[Int] is not a subtype of List[Int] (lists are sequences, but not the other way around).

(3) Elem's third case is not considered because Seq[Int] and List[Int] are not disjoint.

Therefore, the reduction algorithm gets stuck on the second case and the overall type is irreducible. Without disjointness, Elem[Seq[Int]] would reduce to Seq[Int] (via Elem's third case), which would be unsound. For example, the expression elem[Seq[Int]] $\operatorname{List}(1,2,3))$ would have type Seq[Int], but evaluates to the integer 1.

Subsection 3.2 revisits this counterexample in a formal setting. Subsection 4.1 discusses our implementation of disjointness in the Scala compiler.

\section{FORMALIZATION}

In this section, we formally present System FM, an extension of System $F_{<:}$[Cardelli et al. 1994] with pattern matching, opaque classes, and match types. Figure 1 defines FM's syntax and evaluation relation. Figure 2 defines FM's type system, composed of three relations: typing, subtyping, and type disjointness. We discuss differences to System $\mathrm{F}_{<:}$in the following subsections (Subsection 3.1 and 3.2). In Subsection 3.3, we outline a proof of type safety for System FM. In Subsection 3.4, we present an extension of System FM with support for binding pattern variables in type patterns.

\subsection{Classes}

System FM is parametrized by a set of classes $\mathrm{C}$ with class inheritance $\Psi$ and class disjointness $\Xi$. The class inheritance forms a partial order on $C$, that is, it is reflexive, antisymmetric and transitive. The class disjointness is symmetric relation over $\mathrm{C}$. The inheritance and disjointness parameters can be understood as a representation of a hierarchy of Scala traits and classes. For example, trait $\mathrm{C} 1$; class $\mathrm{C} 2$ extends $\mathrm{C} 1$ is represented in $\mathrm{FM}$ as $\Psi=\left\{\left(\mathrm{C}_{1}, \mathrm{C}_{2}\right)\right\} ; \Xi=\{\}$. This representation also models the fact that certain types cannot possibly have common instances. For example, class $\mathrm{C} 3$; class $\mathrm{C} 4$ is represented as $\Psi=\{\} ; \Xi=\left\{\left(\mathrm{C}_{3}, \mathrm{C}_{4}\right),\left(\mathrm{C}_{4}, \mathrm{C}_{3}\right)\right\}$, since Scala disallows multiple class inheritance. Inheritance and disjointness must be consistent in the sense that $(\mathrm{A}, \mathrm{B}) \in \Xi$ implies that there is no class $\mathrm{C}$ such that $(\mathrm{C}, \mathrm{A}) \in \Psi$ and $(\mathrm{C}, \mathrm{B}) \in \Psi$.

Each class in $\mathrm{C}$ gives rise to a constructor (written new $\mathrm{C}$ ), a type (written $\mathrm{C}$ ), and a constructor singleton type (written $\{$ new $\mathrm{C}\}$ ). The type $\mathrm{C}$ denotes all values that inherit $\mathrm{C}$, while the constructor singleton type $\{$ new $C\}$ denotes a single value: C's constructor call. Subtyping between classes is dictated by $\Psi$ via the S-Psi rule.

This parametric approach allows us to model class inheritance as found in object-oriented languages without the need for dedicated syntax for classes and data type definitions. Although our approach might appear simplistic, it can easily model advanced object-oriented features such as multiple inheritance. We discuss the encoding of Scala's types into System FM in Subsection 4.1.

Our type system refers to classes by names and therefore mixes structural and nominal types. Names are useful to give a direct correspondence between runtime tags and compile-time types. As we will see, runtime tags are essential to runtime type testing and play a central role in the evaluation of pattern matching. 
Syntax

\begin{tabular}{|c|c|c|c|}
\hline \multicolumn{2}{|l|}{$t::=$} & \multicolumn{2}{|l|}{$\mathrm{T}::=$} \\
\hline $\mathrm{x}$ & variable & $\mathrm{X}$ & type variable \\
\hline$\lambda \mathrm{x}: \mathrm{T} . \mathrm{t}$ & abstraction & $\mathrm{T} \rightarrow \mathrm{T}$ & type of functions \\
\hline$\lambda \mathrm{X}<: \mathrm{T} . \mathrm{t}$ & type abstraction & $\forall \mathrm{X}<: \mathrm{T} . \mathrm{T}$ & universal type \\
\hline $\mathrm{tt}$ & application & Top & maximum type \\
\hline $\mathrm{t} \mathrm{T}$ & type application & $\mathrm{C}$ & class \\
\hline new C & constructor call & $\{$ new $C\}$ & structor singleton \\
\hline $\mathrm{t} \operatorname{match}\{\overline{\mathrm{x}}$ & or $\mathrm{t}$ match expr. & $\mathrm{T} \operatorname{match}\{\overline{\mathrm{T} \Rightarrow \mathrm{T}}\}$ or $\mathrm{T}$ & match type \\
\hline$:=$ & & $\Gamma::=$ & \\
\hline$\lambda \mathrm{x}: \mathrm{T} . \mathrm{t}$ & abstraction & $\varnothing$ & empty context \\
\hline$\lambda \mathrm{X}<: \mathrm{T} . \mathrm{t}$ & type abstraction & $\Gamma, \mathrm{x}: \mathrm{T}$ & term binding \\
\hline new $\mathrm{C}$ & constructor call & $\Gamma, \mathrm{X}<: \mathrm{T}$ & type binding \\
\hline
\end{tabular}

Evaluation

$$
\begin{aligned}
& \frac{\mathrm{t}_{1} \longrightarrow \mathrm{t}_{1}^{\prime}}{\mathrm{t}_{1} \mathrm{t}_{2} \longrightarrow \mathrm{t}_{1}^{\prime} \mathrm{t}_{2}} \text { (E-App1) } \quad \frac{\mathrm{t}_{2} \longrightarrow \mathrm{t}_{2}^{\prime}}{\mathrm{v}_{1} \mathrm{t}_{2} \longrightarrow \mathrm{v}_{1} \mathrm{t}_{2}^{\prime}} \text { (E-App2) } \quad \frac{\mathrm{t}_{1} \longrightarrow \mathrm{t}_{1}^{\prime}}{\mathrm{t}_{1} \mathrm{~T}_{2} \longrightarrow \mathrm{t}_{1}^{\prime} \mathrm{T}_{2}} \text { (E-TApP) } \\
& \left(\lambda \mathrm{x}: \mathrm{T}_{11} \cdot \mathrm{t}_{12}\right) \mathrm{v}_{2} \longrightarrow\left[\mathrm{x} \mapsto \mathrm{v}_{2}\right] \mathrm{t}_{12} \quad\left(\lambda \mathrm{X}<: \mathrm{T}_{11} \cdot \mathrm{t}_{12}\right) \mathrm{T}_{2} \longrightarrow\left[\mathrm{X} \mapsto \mathrm{T}_{2}\right] \mathrm{t}_{12} \\
& \text { (E-AppAвs) } \\
& \text { (E-TAppTAвs) }
\end{aligned}
$$$$
\begin{gathered}
\frac{\mathrm{t}_{s} \longrightarrow \mathrm{t}_{s}^{\prime}}{\mathrm{t}_{s} \operatorname{match}\left\{\mathrm{x}_{i}: \mathrm{C}_{i} \Rightarrow \mathrm{t}_{i}\right\} \text { or } \mathrm{t}_{d} \longrightarrow} \\
\mathrm{t}_{s}^{\prime} \operatorname{match}\left\{\mathrm{x}_{i}: \mathrm{C}_{i} \Rightarrow \mathrm{t}_{i}\right\} \text { or } \mathrm{t}_{d}
\end{gathered}
$$$$
\frac{\left(\mathrm{C}, \mathrm{C}_{n}\right) \in \Psi \quad \forall m<n .\left(\mathrm{C}, \mathrm{C}_{m}\right) \notin \Psi}{\text { new } \mathrm{C} \text { match }\left\{\mathrm{x}_{i}: \mathrm{C}_{i} \Rightarrow \mathrm{t}_{i}\right\} \text { or } \mathrm{t}_{d} \longrightarrow\left[\mathrm{x}_{n} \mapsto \text { new } \mathrm{C}\right] \mathrm{t}_{n}}(\text { E-MATch2 })
$$$$
\frac{\forall m \cdot\left(\mathrm{C}, \mathrm{C}_{m}\right) \notin \Psi}{\text { new } \mathrm{C} \operatorname{match}\left\{\mathrm{x}_{i}: \mathrm{C}_{i} \Rightarrow \mathrm{t}_{i}\right\} \text { or } \mathrm{t}_{d} \longrightarrow \mathrm{t}_{d}}
$$$$
(\lambda \mathrm{x}: \text { T. t }) \operatorname{match}\left\{\mathrm{x}_{i}: \mathrm{C}_{i} \Rightarrow \mathrm{t}_{i}\right\} \text { or } \mathrm{t}_{d} \longrightarrow \mathrm{t}_{d}
$$$$
(\lambda \mathrm{X}<\mathrm{T} \text {. t }) \operatorname{match}\left\{\mathrm{x}_{i}: \mathrm{C}_{i} \Rightarrow \mathrm{t}_{i}\right\} \text { or } \mathrm{t}_{d} \longrightarrow \mathrm{t}_{d}
$$

Fig. 1. System FM syntax and evaluation rules for a given set of classes $C$ with class inheritance $\Psi$ and class disjointness $\Xi$. Highlights correspond to additions to System $\mathrm{F}_{<:}$. 


\section{Subtyping}

$$
\begin{aligned}
& \Gamma \vdash \mathrm{S}<: \mathrm{S} \quad(\mathrm{S}-\mathrm{REFL}) \\
& \Gamma \vdash \mathrm{S}<\text { : Top } \\
& \Gamma \vdash\{\text { new } \mathrm{C}\}<\text { : } \\
& \frac{\left(\mathrm{C}_{1}, \mathrm{C}_{2}\right) \in \Psi}{\Gamma \vdash \mathrm{C}_{1}<: \mathrm{C}_{2}} \\
& \frac{\Gamma \vdash \mathrm{S}<: \mathrm{U} \quad \Gamma \vdash \mathrm{U}<: \mathrm{T}}{\Gamma \vdash \mathrm{S}<: \mathrm{T}} \text { (S-TrAnS) } \\
& \frac{\Gamma \vdash \mathrm{T}_{1}<: \mathrm{S}_{1} \quad \Gamma \vdash \mathrm{S}_{2}<: \mathrm{T}_{2}}{\Gamma \vdash \mathrm{S}_{1} \rightarrow \mathrm{S}_{2}<: \mathrm{T}_{1} \rightarrow \mathrm{T}_{2}} \text { (S-ArRow) } \\
& \frac{\mathrm{X}<: \mathrm{T} \in \Gamma}{\Gamma \vdash \mathrm{X}<: \mathrm{T}} \\
& \frac{\Gamma, \mathrm{X}<: \mathrm{U}_{1} \vdash \mathrm{S}_{2}<: \mathrm{T}_{2}}{\Gamma \vdash\left(\forall \mathrm{X}<: \mathrm{U}_{1} . \mathrm{S}_{2}\right)<:\left(\forall \mathrm{X}<: \mathrm{U}_{1} \cdot \mathrm{T}_{2}\right)}
\end{aligned}
$$

$$
\frac{\Gamma \vdash \mathrm{T}_{s}<: \mathrm{S}_{n} \quad \forall m<n . \Gamma \vdash \operatorname{disj}\left(\mathrm{T}_{s}, \mathrm{~S}_{m}\right)}{\Gamma \vdash \mathrm{T}_{s} \operatorname{match}\left\{\mathrm{S}_{i} \Rightarrow \mathrm{T}_{i}\right\} \text { or } \mathrm{T}_{d}=:=\mathrm{T}_{n}}
$$

$$
\frac{\forall n . \Gamma \vdash \operatorname{disj}\left(\mathrm{T}_{s}, \mathrm{~S}_{n}\right)}{\Gamma \vdash \mathrm{T}_{s} \operatorname{match}\left\{\mathrm{S}_{i} \Rightarrow \mathrm{T}_{i}\right\} \text { or } \mathrm{T}_{d}=:=\mathrm{T}_{d}}
$$

(S-MATch3/4)

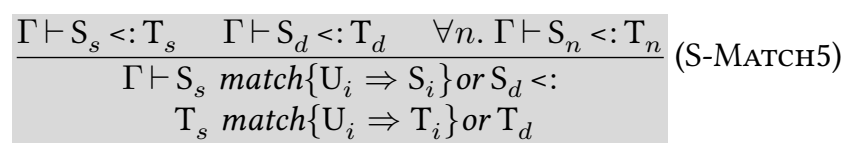

Typing

$$
\begin{aligned}
& \frac{\Gamma, \mathrm{x}: \mathrm{T}_{1} \vdash \mathrm{t}_{2}: \mathrm{T}_{2}}{\Gamma \vdash \lambda \mathrm{x}: \mathrm{T}_{1} \cdot \mathrm{t}_{2}: \mathrm{T}_{1} \rightarrow \mathrm{T}_{2}}(\mathrm{~T}-\mathrm{ABs}) \quad \frac{\Gamma \vdash \mathrm{t}_{1}: \mathrm{T}_{11} \rightarrow \mathrm{T}_{12} \quad \Gamma \vdash \mathrm{t}_{2}: \mathrm{T}_{11}}{\Gamma \vdash \mathrm{t}_{1} \mathrm{t}_{2}: \mathrm{T}_{12}} \text { (T-App) } \\
& \frac{\Gamma, \mathrm{X}<: \mathrm{U}_{1} \vdash \mathrm{t}_{2}: \mathrm{T}_{2}}{\Gamma \vdash \lambda \mathrm{X}<: \mathrm{U}_{1} \cdot \mathrm{t}_{2}: \forall \mathrm{X}<: \mathrm{U}_{1} \cdot \mathrm{T}_{2}} \text { (T-TABs) } \frac{\Gamma \vdash \mathrm{t}_{1}: \forall \mathrm{X}<: \mathrm{T}_{11} \cdot \mathrm{T}_{12} \quad \Gamma \vdash \mathrm{T}_{2}<: \mathrm{T}_{11}}{\Gamma \vdash \mathrm{t}_{1} \mathrm{~T}_{2}:\left[\mathrm{X} \mapsto \mathrm{T}_{2}\right] \mathrm{T}_{12}} \\
& \frac{\mathrm{x}: \mathrm{T} \in \Gamma}{\Gamma \vdash \mathrm{x}: \mathrm{T}}(\mathrm{T}-\mathrm{VAR}) \quad \frac{\Gamma \vdash \mathrm{t}: \mathrm{S} \quad \Gamma \vdash \mathrm{S}<\mathrm{T}}{\Gamma \vdash \mathrm{t}: \mathrm{T}}(\mathrm{T}-\mathrm{SuB}) \quad \Gamma \vdash \text { new } \mathrm{C}:\{\text { new } \mathrm{C}\} \text { (T-Class) }
\end{aligned}
$$

$$
\frac{\Gamma \vdash \mathrm{t}_{s}: \mathrm{T}_{s} \quad \Gamma, \mathrm{x}_{i}: \mathrm{C}_{i} \vdash \mathrm{t}_{i}: \mathrm{T}_{i} \quad \Gamma \vdash \mathrm{t}_{d}: \mathrm{T}_{d}}{\Gamma \vdash \mathrm{t}_{s} \operatorname{match}\left\{\mathrm{x}_{i}: \mathrm{C}_{i} \Rightarrow \mathrm{t}_{i}\right\} \text { or } \mathrm{t}_{d}: \mathrm{T}_{s} \operatorname{match}\left\{\mathrm{C}_{i} \Rightarrow \mathrm{T}_{i}\right\} \text { or } \mathrm{T}_{d}}(\mathrm{~T}-\mathrm{MATCH})
$$

\section{Disjointness}

$$
\begin{array}{cc}
\frac{\left(\mathrm{C}_{1}, \mathrm{C}_{2}\right) \in \Xi}{\Gamma \vdash \operatorname{disj}\left(\mathrm{C}_{1}, \mathrm{C}_{2}\right)} \quad(\mathrm{D}-\mathrm{XI}) & \frac{\left(\mathrm{C}_{1}, \mathrm{C}_{2}\right) \notin \Psi}{\Gamma \vdash \operatorname{disj}\left(\left\{\text { new } \mathrm{C}_{1}\right\}, \mathrm{C}_{2}\right)} \text { (D-Psi) } \\
\frac{\Gamma \vdash \mathrm{S}<: \mathrm{U} \quad \Gamma \vdash \operatorname{disj}(\mathrm{U}, \mathrm{T})}{\Gamma \vdash \operatorname{disj}(\mathrm{S}, \mathrm{T})}(\mathrm{D}-\mathrm{Sub}) & \Gamma \vdash \operatorname{disj}\left(\mathrm{T}_{1} \rightarrow \mathrm{T}_{2}, \mathrm{C}\right)(\mathrm{D}-\text { Arrow }) \\
& \Gamma \vdash \operatorname{disj}\left(\forall \mathrm{X}<: \mathrm{T}_{1}, \mathrm{~T}_{2}, \mathrm{C}\right)(\mathrm{D}-\mathrm{AlL})
\end{array}
$$

Fig. 2. System FM type system for a given set of classes $C$ with class inheritance $\Psi$ and class disjointness $\Xi$. Highlights correspond to additions to System $\mathrm{F}_{<:}$. 


\subsection{Matches}

System FM supports both pattern matching on the term level (match expressions) as well as on the type-level (match types). Matches, both on terms and on types, are composed of a scrutinee, a list of cases and a default expression/type. Each case consists of a type test and a corresponding expression/type. At the term level, a type test consists of an inheritance check against a particular class (this is also known as a typecase [Abadi et al. 1991]). At the type level, a type test corresponds to a subtyping test with a particular type. This disparity reflects the difference between runtime, where type tests are implemented using class tables, and compile time, where types are compared using the type system in its full extent. We discuss the representation of Scala types at runtime in Subsection 4.6.

Throughout this paper, we use the abbreviated syntax $\mathrm{t}_{s} \operatorname{match}\left\{\mathrm{x}_{i}: \mathrm{C}_{i} \Rightarrow \mathrm{t}_{i}\right\}$ or $\mathrm{t}_{d}$ to denote an arbitrary number of cases, that is, $\exists n \in \mathbb{N}$. $\mathrm{t}_{s} \operatorname{match}\left\{\mathrm{x}_{1}: \mathrm{C}_{1} \Rightarrow \mathrm{t}_{1} ; \ldots ; \mathrm{x}_{n}: \mathrm{C}_{n} \Rightarrow \mathrm{t}_{n}\right\}$ or $\mathrm{t}_{d}$.

Match expressions and match types are related by the T-МАтсн typing rule. This rule operates by typing each component of a match expression to then assemble the corresponding match type.

Example A. For example, given two disjoint classes A and B, and an empty class inheritance $(\Psi=\mathrm{Id}, \Xi=\{(\mathrm{A}, \mathrm{B}),(\mathrm{B}, \mathrm{A})\})$; the following function:

$\mathrm{f}=\lambda \mathrm{X}<$ : Top. $\lambda \mathrm{x}: \mathrm{X}$. $\mathrm{x}$ match $\{\mathrm{a}: \mathrm{A} \Rightarrow \mathrm{foo} ; \mathrm{b}: \mathrm{B} \Rightarrow$ bar $\}$ or buzz can be typed precisely as

f : $\forall \mathrm{X}<$ : Top. $\mathrm{X} \rightarrow \mathrm{X}$ match $\{\mathrm{A} \Rightarrow$ Foo; $\Rightarrow$ Bar $\}$ or Buzz

where foo, bar and buzz are expressions with types Foo, Bar and Buzz, respectively.

The cases of a match expression are evaluated sequentially: the scrutinee is checked using the type test of each case, one after the other. The overall expression reduces to the expression that corresponds to the first successful type test (E-MATcH2). When no type test succeeds, the match evaluates to its default expression (E-MAтсH3/4/5). For instance, given the function $\mathrm{f}$ defined in Example A, the expression (f A (new A)) evaluates to foo and (f B $(n e w \mathrm{~B}))$ to bar.

Match Type Reduction. The subtyping relation contains five rules for match type reduction, SMAтсH $1 / 2 / 3 / 4 / 5$. These rules are defined in pairs using the $=:=$ shorthand notation, where $S=:=\mathrm{T}$ means that $\mathrm{S}$ and $\mathrm{T}$ are in a mutual subtyping relation. More precisely, S-MAтcH1/2 in Figure 2 corresponds to two typing rules with identical premises and symmetrical conclusion, and the same goes for S-MATcH3/4.

The typing rules for match type reduction are best explained as generalizations of the evaluation relation. Given a match type $\mathrm{M}=\mathrm{T}_{s} \operatorname{match}\left\{\mathrm{C}_{i} \Rightarrow \mathrm{T}_{i}\right\}$ or $\mathrm{T}_{d}, \mathrm{M}$ reduces to $\mathrm{T}_{i}$ if and only if, for every value $\mathrm{t}_{s}$ in $\mathrm{T}_{s}$, the term level expression $\mathrm{t}_{s} \operatorname{match}\left\{\mathrm{x}_{i}: \mathrm{C}_{i} \Rightarrow \mathrm{t}_{i}\right\}$ or $\mathrm{t}_{d}$ evaluates to $\mathrm{t}_{i}$.

The S-MATch $1 / 2$ rules correspond to the evaluation of a match expression to its $n$th case (EMATCH2):

$$
\frac{\Gamma \vdash \mathrm{T}_{s}<: \mathrm{S}_{n} \quad \forall m<n . \Gamma \vdash \operatorname{disj}\left(\mathrm{T}_{s}, \mathrm{~S}_{m}\right)}{\Gamma \vdash \mathrm{T}_{s} \operatorname{match}\left\{\mathrm{S}_{i} \Rightarrow \mathrm{T}_{i}\right\} \text { or } \mathrm{T}_{d}=:=\mathrm{T}_{n}}
$$

The first premise ensures that the $n$th case type test will succeed for every possible value in the scrutinee type $\mathrm{T}_{s}$. Conversely, the second premise is a disjointness judgment, which ensures that no value in the scrutinee type would result in a successful type test for cases prior to the $n$th case The S-MAтсн3/4 rules correspond to an evaluation to the default case (E-МАтсH2), and require disjointness between the scrutinee type and each type test type:

$$
\frac{\forall n . \Gamma \vdash \operatorname{disj}\left(\mathrm{T}_{s}, \mathrm{~S}_{n}\right)}{\Gamma \vdash \mathrm{T}_{s} \operatorname{match}\left\{\mathrm{S}_{i} \Rightarrow \mathrm{T}_{i}\right\} \text { or } \mathrm{T}_{d}=:=\mathrm{T}_{d}}
$$


Disjointness between two classes can be concluded directly using the D-XI rule which uses the class disjointness $\Xi$. Likewise, disjointness between a constructor singleton type and a class can be concluded directly by inspecting the class inheritance $\Psi$ (D-PsI). Function types and universal types are disjoint from classes as they are inhabited by different values (D-Arrow, D-ALL) and thus will never match. The last disjointness rule, D-SuB, states that if $U$ and $T$ are disjoint, then all subtypes of $\mathrm{U}$ are also disjoint with $\mathrm{T}$.

Example B. We continue developing Example A by showing how match type reduction rules can be used to conclude that ( $\mathrm{f} \mathrm{B}($ new $\mathrm{B}))$ has type Bar. Using T-TApp and T-App, the expression can be typed as follows:

f B (new B) : B match $\{\mathrm{A} \Rightarrow$ Foo; $\mathrm{B} \Rightarrow$ Bar $\}$ or Buzz

Since our example assumes an empty class inheritance and $(A, B) \in \Xi$, the S-MATch1 rule gives:

$\varnothing \vdash \mathrm{B} \operatorname{match}\{\mathrm{A} \Rightarrow$ Foo; $\Rightarrow$ Bar $\}$ or Buzz $<$ : Bar

Finally, using T-SuB we get (f B (new B)) : Bar.

Subtyping and Disjointness. One might wonder what happens if we simplify the match type reduction rules by replacing premises of the form $\Gamma \vdash \operatorname{disj}(\mathrm{T}, \mathrm{C})$ by seemingly equivalent premises of the form $(\mathrm{T}, \mathrm{C}) \notin \Psi$. Unfortunately, the resulting system would be unsound, which can be demonstrated with a counterexample. Let us assume the function $\mathrm{f}$ defined in Example A, adding a new class $\mathrm{E}$ with $\Psi=\{(\mathrm{E}, \mathrm{A}),(\mathrm{E}, \mathrm{B})\}$ and $\Xi=\{\}$. Now, consider the term (f B (new E)). Since we have $\varnothing \vdash \mathrm{E}<$ : B, this function application is well-typed and, given that $(\mathrm{E}, \mathrm{A}) \in \Psi$, evaluates to foo. The term-level and type-level reductions are inconsistent! The unsoundness arises when using $(\mathrm{B}, \mathrm{A}) \notin \Psi$ with the modified S-MAтcH1 rule to wrongly conclude that (f B (new E)) has type Bar. This would result in an inconsistency between types (e:Bar) and evaluation (e $\longrightarrow$ foo), and violate type soundness. In System FM, the match type obtained when typing (f B (new E)) does not reduce since the scrutinee type B is neither disjoint with, nor a subtype of the first pattern type test A. In this case, the unreduced match type is assigned "as is". Unreduced types can appear as the result of programming error, but can also be due to the local irreducibility of a match type. For instance, the body of $\mathrm{f}$ is typed with an unreduced type, as shown in Example A, but that type can later become reducible depending on type variable instantiations.

\subsection{Type Safety}

We show the type safety of System FM through the usual progress and preservation theorems. This section provides an overview of the proof structure and states the involved lemmas and theorems. Detailed proofs are available in the supplementary material of this paper, in two different versions. The first version, [Blanvillain et al. 2021a], is a pen-and-paper proof where System FM is exactly as presented in Figure 2. The second version, [Blanvillain et al. 2021b], is a mechanization of the proof in Coq, using the locally nameless representation by Aydemir et al. [2008] to model variable bindings. Our mechanization uses a simplified representation of match types with exactly one case per match. Matches with multiple cases can be expressed by nesting match types in default cases.

Figure 3 gives an overview of the proof structure by showing implications between the various lemmas and theorems. The basic structure resembles that of System $\mathrm{F}_{<:}$'s standard safety proof from [Pierce 2002]. We continue our presentation by introducing the lemmas and theorems used in our type safety proof.

Preliminary Lemmas. Our proof begins with preliminary technical lemmas: Lemma 3.1 (Permutation), Lemma 3.2 (Weakening), Lemma 3.3 (Strengthening), Lemma 3.4 (Substitution). We omit stating these lemmas as they are entirely standard yet relatively lengthy given that they span 


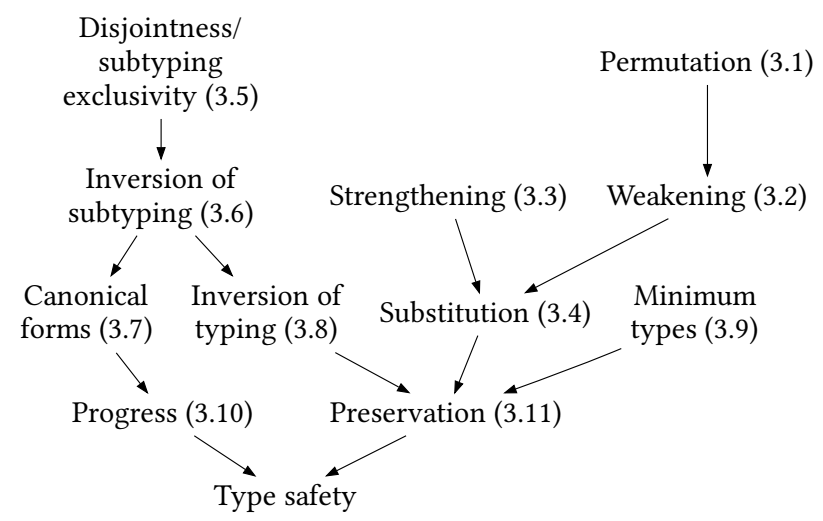

Fig. 3. Structure of the type safety proof. Arrows represent implications between lemmas and theorems.

the three relations of our system: typing, subtyping, and disjointness. As usual, their proofs follow by mutual inductions on derivations.

Disjointness / Subtyping Exclusivity. The following non-standard lemma is necessary to prevent overlap between the S-MAтсH1/2 and S-MAтсH3/4 rules.

LEMMA 3.5 (DisJOINTNESS/SUBTYPING EXCLUSIVITY).

The type disjointness and subtyping relations are mutually exclusive.

If such overlap would be allowed, match types could reduce in several different ways, resulting in an unsound system. We prove Lemma 3.5 by contradiction. Our proof uses a mapping from System FM's types into non-empty subsets of a newly defined set $\mathrm{P}=\{\Lambda, \mathrm{V}\} \cup \mathrm{C}$. Elements of $\mathrm{P}$ can be understood as equivalence classes for FM's types. We show that the subtyping relation in FM corresponds to a subset relation in P, and that the type disjointness relation in FM (disj) corresponds to set disjointness in P. This set-theoretical view lets us conclude the desired result directly. In our Coq mechanization, we axiomatize this lemma and delegate to the pen-and-paper proof.

Inversion of Subtyping. The following Lemma 3.6 allows us to perform inversion on the subtyping relation, which is important to show canonical forms (Lemma 3.7) and inversion of typing (Lemma 3.8). Stating the lemma requires the definition of a new relation denoted $\Gamma \vdash \mathrm{S} \rightleftharpoons \mathrm{T}$, defined in Figure 4. It represents evidence of the mutual subtyping between a match type $\mathrm{S}$ and a type $\mathrm{T}$ with the additional constraint that this evidence was exclusively constructed using pairwise applications of S-MAтch1/2, S-MAтch3/4, and S-Trans in both directions. Intuitively, $\Gamma \vdash \mathrm{S} \rightleftharpoons \mathrm{T}$ is handier than two independent derivations of $\Gamma \vdash \mathrm{S}<$ : $\mathrm{T}$ and $\Gamma \vdash \mathrm{T}<: \mathrm{S}$ because it allows simultaneous induction on both subtyping directions.

LEMMA 3.6 (INVERSION OF SUBTYPING).

(1) If $\Gamma \vdash \mathrm{S}_{s} \operatorname{match}\left\{\mathrm{U}_{i} \Rightarrow \mathrm{S}_{i}\right\}$ or $\mathrm{S}_{d} \rightleftharpoons \mathrm{T}$, then either:

(a) $\Gamma \vdash \mathrm{S}_{s}<: \mathrm{U}_{n}, \forall m<n$. $\Gamma \vdash \operatorname{disj}\left(\mathrm{S}_{s}, \mathrm{U}_{m}\right)$ and $\mathrm{S}_{n}$ is a match type with $\Gamma \vdash \mathrm{S}_{n} \rightleftharpoons \mathrm{T}$,

(b) $\Gamma \vdash \mathrm{S}_{s}<: \mathrm{U}_{n}, \forall m<n$. $\Gamma \vdash \operatorname{disj}\left(\mathrm{S}_{s}, \mathrm{U}_{m}\right)$ and $\mathrm{S}_{n}=\mathrm{T}$,

(c) $\forall n$. $\Gamma \vdash \operatorname{disj}\left(\mathrm{S}_{s}, \mathrm{U}_{n}\right)$ and $\mathrm{S}_{d}$ is a match type with $\Gamma \vdash \mathrm{S}_{d} \rightleftharpoons \mathrm{T}$,

(d) $\forall n . \Gamma \vdash \operatorname{disj}\left(\mathrm{S}_{s}, \mathrm{U}_{n}\right)$ and $\mathrm{S}_{d}=\mathrm{T}$.

(2) If $\Gamma \vdash \mathrm{S}<$ : $\mathrm{X}$, or $\Gamma \vdash \mathrm{S}<$ : $\mathrm{T}$ where $\mathrm{T}$ is a match type with $\Gamma \vdash \mathrm{T} \rightleftharpoons \mathrm{X}$, then either

(a) $\mathrm{S}$ is a match type with $\Gamma \vdash \mathrm{S} \rightleftharpoons \mathrm{Y}$, for some $\mathrm{Y}$, 


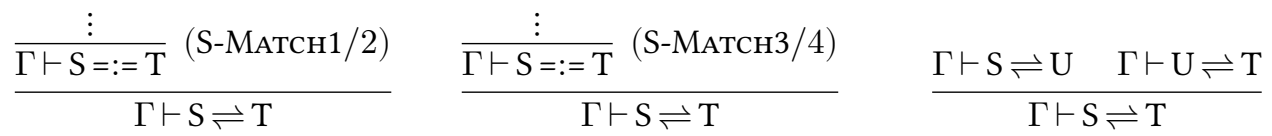

Fig. 4. Definition of the auxiliary relation $\rightleftharpoons$, used to state inversion of subtyping (Lemma 3.6).

(b) $\mathrm{S}$ is a type variable.

(3) If $\Gamma \vdash \mathrm{S}<$ : $\mathrm{T}_{1} \rightarrow \mathrm{T}_{2}$, or $\Gamma \vdash \mathrm{S}<$ : $\mathrm{T}$ where $\mathrm{T}$ is a match type with $\Gamma \vdash \mathrm{T} \rightleftharpoons \mathrm{T}_{1} \rightarrow \mathrm{T}_{2}$, then either

(a) $\mathrm{S}$ is a match type with $\Gamma \vdash \mathrm{S} \rightleftharpoons \mathrm{S}_{1} \rightarrow \mathrm{S}_{2}$, for some $\mathrm{S}_{1}, \mathrm{~S}_{2}$ such that $\Gamma \vdash \mathrm{T}_{1}<: \mathrm{S}_{1}$ and $\Gamma \vdash \mathrm{S}_{2}<$ : $\mathrm{T}_{2}$,

(b) $\mathrm{S}$ is a match type with $\Gamma \vdash \mathrm{S} \rightleftharpoons \mathrm{X}$, for some $\mathrm{X}$,

(c) $\mathrm{S}$ is a type variable,

(d) $\mathrm{S}$ has the form $\mathrm{S}_{1} \rightarrow \mathrm{S}_{2}$ with $\Gamma \vdash \mathrm{T}_{1}<$ : $\mathrm{S}_{1}$ and $\Gamma \vdash \mathrm{S}_{2}<$ : $\mathrm{T}_{2}$.

(4) If $\Gamma \vdash \mathrm{S}<: \forall \mathrm{X}<$ : $\mathrm{U}_{1} \cdot \mathrm{T}_{2}$, or $\Gamma \vdash \mathrm{S}<$ : $\mathrm{T}$ where $\mathrm{T}$ is a match type with $\Gamma \vdash \mathrm{T} \rightleftharpoons \forall \mathrm{X}<$ : $\mathrm{U}_{1} \cdot \mathrm{T}_{2}$, then either

(a) $\mathrm{S}$ is a match type with $\Gamma \vdash \mathrm{S} \rightleftharpoons \forall \mathrm{X}<$ : $\mathrm{U}_{1}$. $\mathrm{S}_{2}$, for some $\mathrm{S}_{2}$ such that $\Gamma, \mathrm{X}<$ : $\mathrm{U}_{1} \vdash \mathrm{S}_{2}<$ : $\mathrm{T}_{2}$,

(b) $\mathrm{S}$ is a match type with $\Gamma \vdash \mathrm{S} \rightleftharpoons \mathrm{X}$, for some $\mathrm{X}$,

(c) $\mathrm{S}$ is a type variable,

(d) $\mathrm{S}$ has the form $\forall \mathrm{X}<: \mathrm{U}_{1}$. $\mathrm{S}_{2}$ and $\Gamma, \mathrm{X}<$ : $\mathrm{U}_{1} \vdash \mathrm{S}_{2}<$ : $\mathrm{T}_{2}$.

The first point of Lemma 3.6 uses the structure of the $\rightleftharpoons$ to provide a form of inversion, which we use to prove each of the subsequent points. In comparison with the corresponding inversion lemma in $\mathrm{F}_{<:}$'s safety proof, the statement and the proof of Lemma 3.6 are longer and more intricate. This difference is inevitable, given that match type reduction rules allow match expressions to be typed as the result of their reduction, which complexifies the inversion.

Similarly to inversion of subtyping, our canonical forms lemma is non-standard in that it uses a disjunction in its premise to account for match types.

LEMMA 3.7 (CANONICAL FORMS).

(1) If $\Gamma \vdash \mathrm{t}: \mathrm{T}$, where either $\mathrm{T}$ is a type variable, or $\mathrm{T}$ is a match type with $\Gamma \vdash \mathrm{T} \rightleftharpoons \mathrm{X}$, then $\mathrm{t}$ is not a closed value.

(2) If $\mathrm{v}$ is a closed value with $\Gamma \vdash \mathrm{v}: \mathrm{T}$ where either $\mathrm{T}=\mathrm{T}_{1} \rightarrow \mathrm{T}_{2}$, or $\mathrm{T}$ is a match type and $\Gamma \vdash$ $\mathrm{T} \rightleftharpoons \mathrm{T}_{1} \rightarrow \mathrm{T}_{2}$, then $\mathrm{v}$ has the form $\lambda \mathrm{x}: \mathrm{S}_{1} \cdot \mathrm{t}_{2}$.

(3) If $\mathrm{v}$ is a closed value with $\Gamma \vdash \mathrm{v}$ : $\mathrm{T}$ where either $\mathrm{T}=\forall \mathrm{X}<: \mathrm{U}_{1} . \mathrm{T}_{2}$, or $\mathrm{T}$ is a match type and $\Gamma$ $\vdash \mathrm{T} \rightleftharpoons \forall \mathrm{X}<: \mathrm{U}_{1} \cdot \mathrm{T}_{2}$, then $\mathrm{v}$ has the form $\lambda \mathrm{X}<: \mathrm{U}_{1} \cdot \mathrm{t}_{2}$.

Proof of Soundness. The remaining proof of soundness is mostly standard. Lemma 3.8 and 3.9 are simple inversions of typing rules. Lemma 3.9 is needed in the proof of preservation to recover subtyping bounds from typing judgments. The proofs proceed by routine induction on derivations.

LEMMA 3.8 (INVERSION OF TYPING).

(1) If $\Gamma \vdash \lambda \mathrm{x}: \mathrm{S}_{1} \cdot \mathrm{s}_{2}: \mathrm{T}$ and $\Gamma \vdash \mathrm{T}<: \mathrm{U}_{1} \rightarrow \mathrm{U}_{2}$, then $\Gamma \vdash \mathrm{U}_{1}<: \mathrm{S}_{1}$ and there is some $\mathrm{S}_{2}$ such that $\Gamma, \mathrm{x}$ : $\mathrm{S}_{1} \vdash \mathrm{s}_{2}: \mathrm{S}_{2}$ and $\Gamma \vdash \mathrm{S}_{2}<: \mathrm{U}_{2}$.

(2) If $\Gamma \vdash \lambda \mathrm{X}<: \mathrm{S}_{1}, \mathrm{~s}_{2}: \mathrm{T}$ and $\Gamma \vdash \mathrm{T}<:\left(\forall \mathrm{X}<: \mathrm{U}_{1}, \mathrm{U}_{2}\right)$, then $\mathrm{U}_{1}=\mathrm{S}_{1}$ and there is some $\mathrm{S}_{2}$ such that $\Gamma, \mathrm{X}<: \mathrm{S}_{1} \vdash \mathrm{s}_{2}: \mathrm{S}_{2}$ and $\Gamma, \mathrm{X}<: \mathrm{S}_{1} \vdash \mathrm{S}_{2}<: \mathrm{U}_{2}$.

LEMMA 3.9 (Minimum TyPes).

(1) If $\Gamma \vdash$ new $\mathrm{C}: \mathrm{T}$ then $\Gamma \vdash\{$ new $\mathrm{C}\}<: \mathrm{T}$.

(2) If $\Gamma \vdash \lambda \mathrm{x}: \mathrm{T}_{1} \cdot \mathrm{t}_{2}: \mathrm{T}$ then there is some $\mathrm{T}_{2}$ such that $\Gamma \vdash \mathrm{T}_{1} \rightarrow \mathrm{T}_{2}<: \mathrm{T}$.

(3) If $\Gamma \vdash \lambda \mathrm{X}<$ : $\mathrm{U}_{1} \cdot \mathrm{t}_{2}: \mathrm{T}$ then there is some $\mathrm{T}_{2}$ such that $\Gamma \vdash \forall \mathrm{X}<$ : $\mathrm{U}_{1} \cdot \mathrm{T}_{2}<$ : $\mathrm{T}$. 
Syntax

$\mathrm{C}::=$

A

B T ground class parametric class

Evaluation

$$
\begin{aligned}
\mathrm{t}::=\ldots & \\
\mathrm{T}::=\ldots & \mathrm{t} \text { match }[\mathrm{X}][\overline{\mathrm{x}: \mathrm{C} \Rightarrow \mathrm{t}}\} \text { or } \mathrm{t} \text { match expr. } \\
& \mathrm{T} \text { match }[\mathrm{X}]\{\overline{\mathrm{T} \Rightarrow \mathrm{T}}\} \text { or } \mathrm{T} \quad \text { match type }
\end{aligned}
$$

$$
\begin{gathered}
\frac{\left(\mathrm{C},[\mathrm{X} \mapsto \mathrm{U}] \mathrm{C}_{n}\right) \in \Psi \quad \forall m<n . \forall \mathrm{T} .\left(\mathrm{C},[\mathrm{X} \mapsto \mathrm{T}] \mathrm{C}_{m}\right) \notin \Psi}{\text { new } \mathrm{C} \text { match }[\mathrm{X}]\left\{\mathrm{x}_{i}: \mathrm{C}_{i} \Rightarrow \mathrm{t}_{i}\right\} \text { or } \mathrm{t}_{d} \longrightarrow[\mathrm{X} \mapsto \mathrm{U}]\left[\mathrm{x}_{n} \mapsto \text { new } \mathrm{C}\right] \mathrm{t}_{n}}(\text { BE-Match2) } \\
\frac{\forall m . \forall \mathrm{T} .\left(\mathrm{C},[\mathrm{X} \mapsto \mathrm{T}] \mathrm{C}_{m}\right) \notin \Psi}{\text { new } \mathrm{C} \text { match }[\mathrm{X}]\left\{\mathrm{x}_{i}: \mathrm{C}_{i} \Rightarrow \mathrm{t}_{i}\right\} \text { or } \mathrm{t}_{d} \longrightarrow \mathrm{t}_{d}}
\end{gathered}
$$

Subtyping

$$
\begin{gathered}
\frac{\Gamma, \mathrm{X}<: \mathrm{U} \vdash \mathrm{T}_{s}<: \mathrm{S}_{n} \quad \forall m<n . \Gamma, \mathrm{X}<: \mathrm{Top} \vdash \operatorname{disj}\left(\mathrm{T}_{s}, \mathrm{~S}_{m}\right)}{\Gamma \vdash \mathrm{T}_{s} \text { match }[\mathrm{X}]\left\{\mathrm{S}_{i} \Rightarrow \mathrm{T}_{i}\right\} \text { or } \mathrm{T}_{d}=:=[\mathrm{X} \mapsto \mathrm{U}] \mathrm{T}_{n}} \\
\frac{\forall n . \Gamma, \mathrm{X}<: \mathrm{Top} \vdash \operatorname{disj}\left(\mathrm{T}_{s}, \mathrm{~S}_{n}\right)}{\Gamma \vdash \mathrm{T}_{s} \text { match }[\mathrm{X}]\left\{\mathrm{S}_{i} \Rightarrow \mathrm{T}_{i}\right\} \text { or } \mathrm{T}_{d}=:=\mathrm{T}_{d}} \\
\frac{\Gamma \vdash \mathrm{S}_{s}<: \mathrm{T}_{s} \quad \Gamma \vdash \mathrm{S}_{d}<: \mathrm{T}_{d} \quad \forall n . \Gamma, \mathrm{X}<: \mathrm{Top} \vdash \mathrm{S}_{n}<: \mathrm{T}_{n}}{\Gamma \vdash \mathrm{S}_{s} \text { match }[\mathrm{X}]\left\{\mathrm{U}_{i} \Rightarrow \mathrm{S}_{i}\right\} \text { or } \mathrm{S}_{d}<:} \\
\mathrm{T}_{s} \text { match }[\mathrm{X}]\left\{\mathrm{U}_{i} \Rightarrow \mathrm{T}_{i}\right\} \text { or } \mathrm{T}_{d}
\end{gathered}
$$

Typing

$$
\frac{\Gamma \vdash \mathrm{t}_{s}: \mathrm{T}_{s} \quad \Gamma, \mathrm{X}<: \text { Top }, \mathrm{x}_{i}: \mathrm{C}_{i} \vdash \mathrm{t}_{i}: \mathrm{T}_{i} \quad \Gamma \vdash \mathrm{t}_{d}: \mathrm{T}_{d}}{\Gamma \vdash \mathrm{t}_{s} \text { match }[\mathrm{X}]\left\{\mathrm{x}_{i}: \mathrm{C}_{i} \Rightarrow \mathrm{t}_{i}\right\} \text { or } \mathrm{t}_{d}: \mathrm{T}_{s} \text { match }[\mathrm{X}]\left\{\mathrm{C}_{i} \Rightarrow \mathrm{T}_{i}\right\} \text { or } \mathrm{T}_{d}} \text { (BT-MATcH) }
$$

Fig. 5. System FMB syntax, evaluation and typing rules for a given set of ground classes A, set of parametric classes B, class inheritance $\Psi$, and class disjointness $\Xi$. Highlights correspond to changes made to System FM.

With these lemmas in hand, the proofs of progress and preservation are straightforward.

Theorem 3.10 (Progress).

If $\mathrm{t}$ is a closed, well-typed term, then either $\mathrm{t}$ is a value or there is some $\mathrm{t}^{\prime}$ such that $\mathrm{t} \longrightarrow \mathrm{t}^{\prime}$.

Theorem 3.11 (Preservation).

If $\Gamma \vdash \mathrm{t}: \mathrm{T}$ and $\mathrm{t} \longrightarrow \mathrm{t}^{\prime}$ then $\Gamma \vdash \mathrm{t}^{\prime}: \mathrm{T}$.

\subsection{Type Binding Extension}

In this section, we present System FMB, an extension of System FM with support for binding pattern variables in type patterns. FMB is parametrized by two sets of classes, A and B, representing non-parametric and parametric classes, respectively. A parametric class, written $\mathrm{B} T$, takes exactly one type parameter ${ }^{1}$. We redefine $C$ to be a syntactic object defined as $C::=A \mid B$ T . The class inheritance $\Psi$ and class disjointness $\Xi$ remain as binary relations on C. A generic instantiation in 
the class hierarchy is represented as an element of $\Psi$, for example, A1 extends B2[A3] is represented as $\left(\mathrm{A}_{1}, \mathrm{~B}_{2} \mathrm{~A}_{3}\right) \in \Psi$. Generic inheritance is represented using multiple entries in $\Psi$, for example, $\mathrm{B} 1[\mathrm{~T}]$ extends $\mathrm{B} 2[\mathrm{~T}]$ is represented as $\forall \mathrm{T}$. $\left(\mathrm{B}_{1} \mathrm{~T}, \mathrm{~B}_{2} \mathrm{~T}\right) \in \Psi$. This approach allows us to reuse most of FM's definitions. Indeed, our formal development treats $C, \Psi$, and $\Xi$ as mathematical objects, and is compatible with FMB's new definition of classes.

In Figure 5, we define System FMB syntax and rules, where changes to System FM are highlighted in gray. FMB's new syntax for match expressions and match types adds a pattern variable to each construct. In $\mathrm{t}_{s}$ match $[\mathrm{X}]\left\{\mathrm{x}_{i}: \mathrm{C}_{i} \Rightarrow \mathrm{t}_{i}\right\}$ or $\mathrm{t}_{d}$, the pattern variable $\mathrm{X}$ is available to bind type parameters in $\mathrm{C}_{i}$ patterns.

The definitions of S-MAтсн3/4, S-MAтсн5, and T-МАтсH require minor adjustments to account for the pattern variable in typing contexts. Note that pattern variables appear in contexts unconditionally, regardless of whether or not those variables are used in the corresponding patterns.

In the new subtyping rule for non-default match reduction, BS-MAтсH $1 / 2$, the first premise instantiates the pattern variable $\mathrm{X}$ to some type $\mathrm{U}$ such that the scrutinee type is a subtype of the $n$th pattern:

$$
\frac{\Gamma, \mathrm{X}<: \mathrm{U} \vdash \mathrm{T}_{s}<: \mathrm{S}_{n} \quad \forall m<n . \Gamma, \mathrm{X}<: \mathrm{Top} \vdash \operatorname{disj}\left(\mathrm{T}_{s}, \mathrm{~S}_{m}\right)}{\Gamma \vdash \mathrm{T}_{s} \text { match }[\mathrm{X}]\left\{\mathrm{S}_{i} \Rightarrow \mathrm{T}_{i}\right\} \text { or } \mathrm{T}_{d}=:=[\mathrm{X} \mapsto \mathrm{U}] \mathrm{T}_{n}}
$$

Here $\mathrm{U}$ is completely unconstrained: any instantiation of $\mathrm{X}$ such that $\mathrm{T}_{s}<: \mathrm{S}_{n}$ would be admissible. The disjointness judgments use a weaker upper bound for $\mathrm{X}$ than the subtyping judgment $(\mathrm{X}<$ : Top instead of $\mathrm{X}<\mathrm{U} \mathrm{U}$ ). This is because the scrutinee type must be shown disjoint with non-matching pattern types for every possible instantiation of X. In an algorithmic system, $\mathrm{U}$ would be computed during type inference by constraint solving.

The new evaluation rule for non-default match reduction uses a similar mechanism: it looks for the first case where the pattern variable can be instantiated such that the scrutinee inherits the corresponding pattern:

$$
\frac{\left(\mathrm{C},[\mathrm{X} \mapsto \mathrm{U}] \mathrm{C}_{n}\right) \in \Psi \quad \forall m<n . \forall \mathrm{T} .\left(\mathrm{C},[\mathrm{X} \mapsto \mathrm{T}] \mathrm{C}_{m}\right) \notin \Psi}{\text { new } \mathrm{C} \text { match }[\mathrm{X}]\left\{\mathrm{x}_{i}: \mathrm{C}_{i} \Rightarrow \mathrm{t}_{i}\right\} \text { or } \mathrm{t}_{d} \longrightarrow[\mathrm{X} \mapsto \mathrm{U}]\left[\mathrm{x}_{n} \mapsto \text { new } \mathrm{C}\right] \mathrm{t}_{n}}
$$

The second premise rules out non-matching cases with a universal quantifier ranging over all types. A concrete implementation would certainly opt for a more efficient approach, for instance by implementing $\Psi$ as a lookup table.

Example C. Consider the following class hierarchy with two ground classes: Char and String, and a single parametric class List, such that String extends List Char:

$$
\begin{array}{rlrl}
\mathrm{A} & =\{\text { Char }, \text { String }\} & \mathrm{B} & =\{\text { List }\} \\
\Psi & =\{(\text { String, List Char })\} \cup \mathrm{Id} & \Xi & =\{\} \\
\mathrm{f} & =\lambda \mathrm{x}: \text { Top. } \mathrm{x} \text { match }[\mathrm{X}]\{\mathrm{xs}: \text { List } \mathrm{X} & \Rightarrow \text { foo }\} \text { or bar }
\end{array}
$$

The function $\mathrm{f}$ matches its arguments against the List $\mathrm{X}$ pattern, where $\mathrm{X}$ is a pattern variable. We examine the evaluation of two applications of $\mathrm{f}$ :

(1) $\mathrm{f}$ (new String) matches against List $\mathrm{X}$ with $\mathrm{X}=$ Char and evaluates to $[\mathrm{X} \mapsto$ Char $][\mathrm{X} \mapsto$ new String]foo via BE-MATch2 (since (String, List Char) $\in \Psi$ ).

\footnotetext{
${ }^{1}$ The restriction to a single type parameter is for presentation purposes. Both System FMB's type system and type-safety proof can easily be adapted to support a variable number of binding variables.
} 
(2) $\mathrm{f}$ (new List Top) also matches List $\mathrm{X}$, this time with $\mathrm{X}=$ Top, and evaluates to $[\mathrm{X} \mapsto$ Top $][\mathrm{x} \mapsto$ new List Top]foo via BE-MATch2. Here (List Top, List Top) $\in \Psi$ follows from $\Psi$ 's reflexivity.

We established the type safety of System FMB by adapting System FM's pen-and-paper proof. The required changes are lengthy, but relatively uninteresting; it boils down to additional bookkeeping of pattern variables in contexts. The main takeaway from FMB's type safety is that the proof does not require additional constraints on type $\mathrm{U}$ in BS-MATCH1/2. As a result, algorithmic implementations are free to use any mechanism to come up with instantiations of pattern variables.

\section{IMPLEMENTATION}

Match types are implemented in Dotty, the reference compiler for Scala 3. This section explains how our implementation relates to the formalization presented in Section 3.

In the compiler, match-type reduction happens during subtyping, just like in System FM. In order for subtyping to remain algorithmic, match type reduction rules are never used to introduce new match types, but only to simplify the ones present in the original program. The reduction algorithm closely follows the typing rules of Section 3 . The scrutinee type is compared with each pattern sequentially. If the scrutinee is a subtype of the first pattern type, the match type reduces. Otherwise, if the scrutinee can be shown to be disjoint with the first pattern type, the algorithm proceeds to the next pattern. If the algorithm reaches a pattern where neither subtyping nor disjointness can be concluded, the reduction is aborted and the match type remains unreduced.

\subsection{Disjointness in Scala}

Separate compilation is the biggest obstacle to concluding that two types are disjoint. Indeed, in Scala, all traits and classes are extensible by default. Because Scala programs are compiled with an open-world assumption, it is common for types to be effectively disjoint in the current compilation unit, but due to potential extensions in future compilations, the compiler must stay conservative.

Separate compilation is the reason why our formalization requires two different parameters to describe its class hierarchy. One particular instantiation of System FM can be thought of as a model of Scala's type system for a particular compilation unit, where $C$ represents the set of classes declared so far. The class inheritance, $\Psi$, remains valid for all subsequent compilation units: new class definitions do not alter the inheritance between previously defined classes. However, the inheritance parameter $(\Psi)$ is, on its own, not sufficient to conclude that two classes are disjoint: new class definitions can introduce new overlaps between existing classes. For this reason, our formalization uses a separate parameter to describe class disjointness $(\Xi)$. To account for separate compilation, $\Xi$ should only contain pairs of classes which would remain disjoint despite potential additions to the current set of classes.

Thankfully, Scala provides several ways to restrict extensibility. The sealed and final annotations on traits and classes directly restrict the extensibility of annotated types: sealed types can only be extended in the same file as its declaration, thereby providing a way to enumerate all the children of a type. Thus, disjointness of sealed traits and classes can be computed recursively by iterating over all the possible subtypes of that type. The main distinction between traits and classes is that a class can extend at most one superclass. This property allows the compiler to assert that classes are disjoint with a simple check: given two classes $\mathrm{A}$ and $\mathrm{B}$, if neither $\mathrm{A}<$ : $\mathrm{B}$ nor $\mathrm{B}<$ : $\mathrm{A}$, then no class could possibly extend both $\mathrm{A}$ and $\mathrm{B}$, and those two types are disjoint.

As an example, consider the following Scala definitions (left-hand side), and the corresponding instantiation of System FM (right-hand side): 


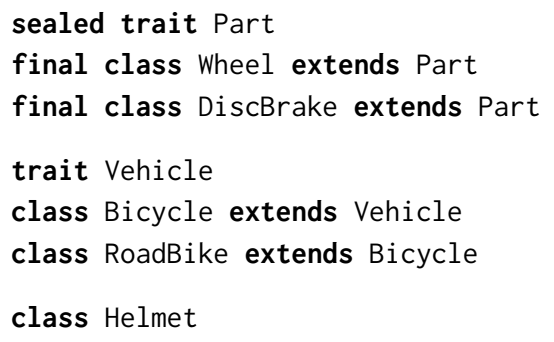

$$
\begin{aligned}
\mathrm{C}=\{ & \mathrm{P}, \mathrm{W}, \mathrm{D}, \mathrm{V}, \mathrm{B}, \mathrm{R}, \mathrm{H}\} \\
\Psi=\{ & (\mathrm{W}, \mathrm{P}),(\mathrm{D}, \mathrm{P}),(\mathrm{B}, \mathrm{V}),(\mathrm{R}, \mathrm{B}),(\mathrm{R}, \mathrm{V})\} \\
\Xi=\{ & (\mathrm{P}, \mathrm{V}),(\mathrm{P}, \mathrm{B}),(\mathrm{P}, \mathrm{R}),(\mathrm{P}, \mathrm{H}) \\
& (\mathrm{W}, \mathrm{V}),(\mathrm{W}, \mathrm{B}),(\mathrm{W}, \mathrm{R}),(\mathrm{W}, \mathrm{H}), \\
& (\mathrm{D}, \mathrm{V}),(\mathrm{D}, \mathrm{B}),(\mathrm{D}, \mathrm{R}),(\mathrm{D}, \mathrm{H}), \\
& (\mathrm{W}, \mathrm{D}),(\mathrm{B}, \mathrm{H}),(\mathrm{R}, \mathrm{H})\}
\end{aligned}
$$

The classes and inheritance relation are practically isomorphic between the two representation: Scala classes have a one-to-one correspondence to their FM counterparts (abbreviated with initials) and the inheritance only contains an additional entry for R and V, obtained by transitivity ( $\Psi$ 's reflexivity and $\Xi$ 's symmetry are omitted for brevity).

$\mathrm{P}$ is declared sealed, meaning that no additional parts can be defined outside of this compilation unit. As a result, we can enumerate all parts to conclude that none are vehicles and $(\mathrm{P}, \mathrm{V}) \in \Xi$. Note that this would not be the case if either W or D was declared non-final, since extending those classes would indirectly create new parts. $\mathrm{B}$ and $\mathrm{H}$ are both classes that do not inherit each other, which implies that $(\mathrm{B}, \mathrm{H}) \in \Xi$ given that Scala classes can extend at most one class. $V$ and $\mathrm{H}$, however, cannot be concluded disjoint in those definitions. If that turns out to be a desirable property, disjointness could easily be obtained by sealing $\mathrm{V}$ or finalizing $\mathrm{H}$.

\subsection{Empty Types}

An important limitation of System FM compared to Scala's type system is that it does not support empty types. The bottom of Scala's subtyping lattice, called Nothing, provides a direct way to refer to empty sets of values. Intersection types also provide a way to construct uninhabited types given that Scala does not forbid intersecting two disjoint types. Empty types are problematic for the match type reduction algorithm as they break the fundamental assumption that two types cannot be both disjoint and subtypes (Lemma 3.5). To account for this, our implementation uses an additional inhabitance check on scrutinee types before attempting any reduction.

To show why empty types are problematic, we can construct an example where, in the absence of an inhabitance check, the same match type could be reduced differently in two different contexts:

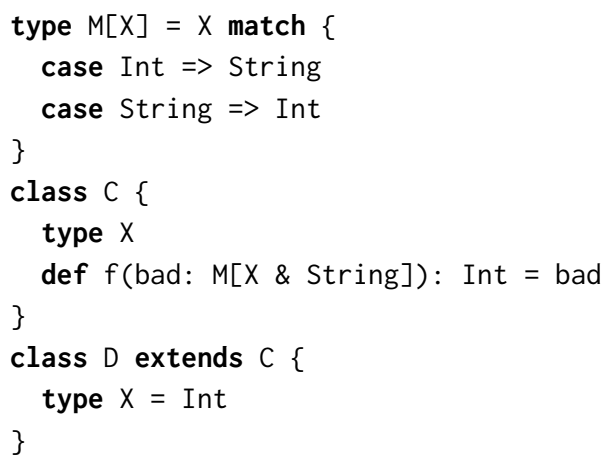

In this example, the definition of $f$ in $C$ type-checks because $X \&$ String and Int are disjoint (since String and Int are disjoint) and M[X \& String] reduces to Int (M's second case applies). Class D refines the definition of $C$ by giving concrete definition of $X$. The unsoundness manifests itself in the body of class D, where X \& String is a subtype of Int and M[X \& String] reduces to String (M's first 
case applies). There, it is possible to call the function $f$ with a string argument, which would result in a runtime error. Checking for scrutinee inhabitance prevents this class of errors. In this example, it would prevent M[Int \& String] from reducing given that Int \& String is not inhabited.

\subsection{Null Values}

In Scala 3, null values no longer inhabit every type: nullable types require explicit annotations of the form A | Null [Nieto et al. 2020]. Our implementation of subtyping and disjointness handles union types and therefore needs no particular treatment of null values.

\subsection{Variance}

Scala supports variance annotations on type parameters of higher-kinded types. These annotations allow programmers to specify how the subtyping of annotated parameters influences the subtyping of the higher-kinded type. For instance, type $\mathrm{F}[+\mathrm{T}]$ defines a type $\mathrm{F}$ that is covariant in its first type parameter, meaning that $\mathrm{T} 1<: \mathrm{T} 2$ implies $\mathrm{F}[\mathrm{T} 1]<: \mathrm{F}[\mathrm{T} 2]$. Contravariance, written type $\mathrm{G}[-\mathrm{T}]$, has the opposite meaning: T1 <: T2 implies G[T2] <: G[T1].

It would appear that co- and contravariant types are always overlapping, given that, for all types X, F[Nothing $]<: F[X]$ and G[Any $]<: G[X]$ (where Nothing and Any are Scala's bottom and top types). However, in the case of covariant parameters, an exception can be made when the said type parameter corresponds to a field or a constructor parameter: the Nothing instantiation can be ruled out because no runtime program can produce a value of that type.

Scala tuples, for example, fall into this category. Tuple2, the class for pairs, is defined as follows: case class Tuple2[+T1, +T2](_1: T1, _2: T2)

Given two instantiations of this this type, Tuple2[Int, X] and Tuple2[String, X], although Tuple2[ Nothing, $X]$ is a subtype of both, there is no runtime value of type Tuple2[ Nothing, $X]$ (since Nothing is uninhabited), and, as a result, those two types are disjoint. Our disjointness algorithm implements this kind of reasoning to conclude disjointness in the presence of covariant type parameters.

\subsection{Pattern Matching Exhaustivity}

The Scala compiler checks for pattern matching exhaustivity to prevent runtime exceptions caused by missing cases. Exhaustivity checking uses static knowledge about the class hierarchy (such as the sealed and final annotations) to check that every value in the scrutinee type is covered by the pattern clauses [Liu 2016]. Non-exhaustive patterns are compiled with an additional "catch-all" case which throws a runtime exception. System FM uses default cases as a replacement for systematic exhaustivity checks or runtime exceptions.

\subsection{Types at Runtime}

Scala's primary platform is the Java virtual machine (JVM). On the JVM, Scala is compiled using partial erasures, where parts of types are preserved and translated to the JVM's type systems, and other parts are erased [Schinz 2005]. For instance, ground classes are compiled directly to JVM classes, but type parameters and type variables are erased and replaced by their bounds.

Erasure directly affects the type tests that can be performed at runtime. For example, while case $x s$ : List[Int] $\Rightarrow$ is a syntactically valid pattern, it will lead to a compiler warning since the Int type parameter is eliminated by erasure and cannot be checked at runtime.

This restriction is reflected in our formalism by the difference between the evaluation rules for match expressions and the reduction rules for match types: evaluation is limited to inheritance checks on statically defined classes $\left(\left(\mathrm{C}, \mathrm{C}_{n}\right) \in \Psi\right.$ in E-MATcH2/3), as opposed to the match type 
reduction rules which are defined using the subtyping and type disjointness relations $\left(\Gamma \vdash \mathrm{T}_{s}<: \mathrm{S}_{n}\right.$ and $\Gamma \vdash \operatorname{disj}\left(\mathrm{T}_{s}, \mathrm{~S}_{m}\right)$ in S-MATch1/2/3/4).

In System FMB (Subsection 3.4), match expressions support two sorts of parametric patterns: they can be either instantiated ( $m a t c h\{x s:$ List Int $\}$, where Int $\in A$ ), or use a binding pattern variable (match $[\mathrm{X}]\{\mathrm{xs}:$ List $\mathrm{X}\}$, where $\mathrm{X}$ is a pattern variable). In this sense, System FMB is more expressive than Scala, where instantiated patterns are not available at the term level due to type erasure.

\subsection{Non-Termination}

Unlike our calculus, the Scala implementation also allows match types to be defined recursively. Recursive match types can cause subtyping checks to loop indefinitely. Our implementation does not check match types for termination, as any such check would necessarily limit expressiveness or convenience. Instead, we detect divergence during match type reduction using a fuel mechanism. The compiler is given an initial amount of fuel, which is consumed one unit at a time on every reduction step. If the compiler runs out of fuel, the reduction is aborted with a "recursion limit exceeded" error. The current implementation uses a fixed amount of initial fuel. Although this seems to be sufficient for most practical purposes, we plan on making it configurable. This mechanism is completely standard and already used in other programing languages with unbounded recursion at the type level [Eisenberg 2016; Eisenberg et al. 2014; Sjoberg 2015].

\subsection{Inference}

System FM's type rules enable any match expression to be typed as a match type, but the situation is different in the full Scala language. Pattern matching in Scala supports many sorts of patterns [Emir et al. 2007], most of which do not have a match type counterpart. Furthermore, typing match expressions as match types is not enabled by default in order to preserve backward compatibility. Instead, explicit type annotations must be provided.

\subsection{Caching}

Scala's type-checking algorithm makes heavy use of caching to improve its performance. Special care must be taken when caching the result of match type reduction, given that the subtyping and disjointness checks are context-dependent. Our implementation uses a context-aware cache for match types that automatically invalidates reduction results when match types are reduced in new contexts. An example where naive caching would be incorrect can be found in Subsection 6.4.

\subsection{Size of the Implementation}

In terms of lines of code, our match type implementation is a relatively modest addition to the Scala compiler: the overall changes amount to around 1500 lines (excluding tests and documentation).

\section{CASE STUDY: SHAPE-SAFE NUMPY}

In this section, we present a case study to show how match types can be used to express complex type constraints, which in turn can prevent certain programming errors at compile time. To this end, we outline the type-level implementation of a library for multidimensional arrays which mimics the NumPy API [Harris et al. 2020]. The goal of our library is to provide a shape-safe interface for manipulating $n$-dimensional arrays (abbreviated ndarrays), where array shapes and indices are checked for errors at compile-time rather than at runtime. Shape and indexing errors in ndarrays is a widely acknowledged problem [Barham and Isard 2019; Rush 2019], and several solutions have already been proposed, notably in the form of libraries that rely on type-level programming [Chen 2017; Huang et al. 2021]. Our library uses match types to provide a shape-safe NumPy-like interface.

Proc. ACM Program. Lang., Vol. 6, No. POPL, Article 37. Publication date: January 2022. 
Scala programmers have a long history of using ad-hoc solutions for type-level programming [Blanvillain et al. 2021c; Pilquist and Scodec open-source contributors 2021; Sabin and Shapeless open-source contributors 2021]. These solutions have several downsides, such as being slow to compile and cumbersome to use. Match types aim at simplifying type-level programming by providing first-class language support. We believe that the approach presented in this case study is an improvement over the status quo because it does not use metaprogramming or convoluted implicit-based encodings to express type-level operations.

\subsection{Shape Errors in Python}

In the example Python code below, the img_batch ndarray is a batch of 25 randomly generated RGB images of size $256 \times 256$. The code aims to compute a vector of length 25 containing the average grayscale color of each image in the batch, and then create a square $5 \times 5$ image of the average grayscale colors. However, this code contains a shape error and will throw an error at runtime.

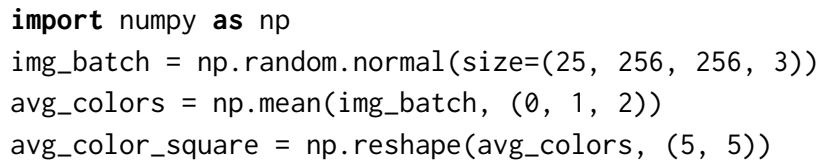

The error is in the call to np.mean, which takes as argument a list of axes to reduce along. Unfortunately, the arguments to np.mean are off-by-one and result in a vector of length 3 (img_batch's last dimension) instead of the intended length of 25 (img_batch's first dimension); avg_color thus contains the average RGB color of the batch instead of the average grayscale color for each image. The reshape operation will then fail at runtime, as it cannot reshape a 3-element vector into a 25 -element matrix. This error can be difficult to spot, given that NumPy's interface for reducing along multiple axes is index-based. Runtime errors like this one can be particularly frustrating when they occur late in a long-running computation.

In the remainder of this section, we show how match types can be used to prevent this class of error. After a preliminary introduction of singleton types (Subsection 5.2), we introduce ndarray shapes at the type level using HLists (Subsection 5.3). In Subsection 5.4, we show type-level implementations of the np.mean and np. reshape operations using match types. Finally, we show how this newly defined API can detect and report the error from our original Python example.

\subsection{Singleton Types}

Scala supports singleton types, which are types inhabited by a single value [Leontiev et al. 2014]. For instance, the singleton type 1 denotes the type containing the integer value 1 . The Scala standard library contains several predefined match types to perform arithmetic operations at the type level. For instance, type $+[A<:$ Int,$B<$ : Int $]$ represents the addition of integer singleton types. Internally, the compiler is special-cased to implement these arithmetic operations using constant folding. Representing numbers with singleton types is desirable for practical purposes, but not absolutely necessary for this case study (for instance, Peano numerals could be used instead).

\subsection{Type-Level Array Shape}

The shape of an ndarray is a list of dimension lengths; we say that the shape $\left(\mathrm{a}_{1}, \mathrm{a}_{2}, \ldots, \mathrm{a}_{n}\right)$ has $n$ dimensions. For instance, a three-by-four matrix is a two-dimensional ndarray of shape $(3,4)$. We represent the shape of an ndarray at the type level using a heterogeneous type list, or HList for 
short [Kiselyov et al. 2004]. We define an HList called Shape as an ADT with two constructors ${ }^{2}$, \#: and $\varnothing$.

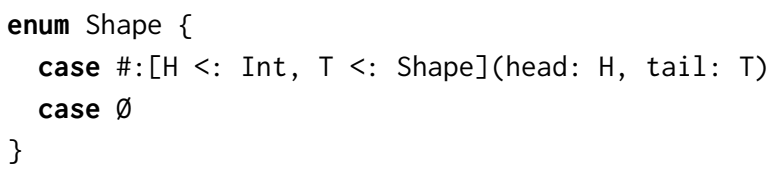

This data type definition allows us to write lists of dimension sizes, both at the term level as

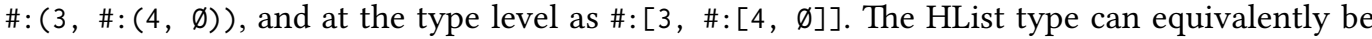
written with \#: in infix notation, as 3 \#: 4 \#: $\varnothing$.

To represent ndarrays, we define the NDArray type. This type is indexed by the ndarray element type $(\mathrm{T})$, and by the ndarray shape (S), represented as an HList:

trait NDArray[T, $\mathrm{S}<$ : Shape]

The goal of our presentation is to define type-safe operations on NDArrays. Since our focus is on the type-level, we do not include value-level counterparts to T and S in the definition of NDArray, but this would be necessary in a complete implementation.

To construct ndarrays, we define random_normal, which creates an ndarray of a given shape, where all elements are random Floats: ${ }^{3}$

def random_normal[S <: Shape](shape: S): NDArray[Float, S] = ???

\subsection{Computation on Shapes with Match Types}

Encoding the types and shapes of ndarrays in the types allows us to readily provide type- and shape-safety for simple ndarray operations. For instance, the element-wise Hadamard product, written as np.multiply $(x, y)$, requires the $x$ and $y$ ndarrays to have the same shape and element types. This constraint does not require any match types, but can simply be expressed as:

def multiply[T, $\mathrm{S}<$ : Shape](x: NDArray $[\mathrm{T}, \mathrm{S}], \mathrm{y}$ : $\operatorname{NDArray}[\mathrm{T}, \mathrm{S}]$ ): $\operatorname{NDArray}[\mathrm{T}, \mathrm{S}]=$ ???

However, we will need the additional expressiveness of match types to implement more complex constraints on array shapes, such as for reshapes (5.4.1) and reductions (5.4.2).

5.4.1 Reshape. An operation commonly used in NumPy is np. reshape, which changes the shape of an ndarray, but does not change its values. A restriction imposed by the NumPy API is that the output shape must have the same number of elements as the input shape. The number of elements of a shape is the product of the sizes of its dimensions; an ndarray of shape 2 \#: 3 \#: 4 \#: $\varnothing$ has $2 \times 3 \times 4=24$ elements. Note that an ndarray of shape $\varnothing$ is a scalar, and thus has a single element. This can be naturally expressed with a match type:

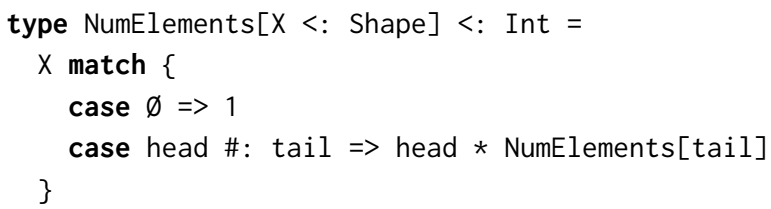

\footnotetext{
${ }^{2}$ In the interest of clarity, our presentation omits type bounds that are necessary to guide type inference, for example in the definition of the Shape data type.

${ }^{3}$ This snippet uses the triple question mark operator, Scala's standard notation for missing or omitted implementations.
} 
To restrict reshaping to be applicable only on valid shapes, the type system must support encoding type equality constraints. For this, we make use of Scala's implicit parameters, and of the =: = type equality constraint that is a part of Scala's standard library.

def reshape[T, From <: Shape, To <: Shape](array: NDArray[T, From], newshape: To)

(implicit ev: NumElements[From] =:= NumElements[To]): NDArray[T, To] = ???

With this definition of reshape, the compiler will only accept a usage of reshape if it is able to prove that the number of elements in the old shape is the same as in the new shape. This example illustrates how match types can be used in concert with existing features like singleton types and implicit resolution to express powerful constraints.

5.4.2 Reduction. The NumPy API provides a variety of functions to reduce along a set of axes of an ndarray, such as np. mean (ndarray, axes) or np.var(ndarray, axes). The axes parameter is a list of indices of dimensions, listing exactly those dimensions that will no longer be present in the output ndarray. The dimension indices can be unordered and repeated, and out-of-bounds indices result in an error. In the Python API, passing the None value instead of a list of axes reduces along all axes, meaning that the operation returns a scalar. Note that this is the opposite of passing $\emptyset$, which means that we reduce over no axes (effectively a no-op).

This behavior is more complex than the previous examples, but can still be described accurately by a match type. We use a match type called Reduce to compute the return shape of the operation.

def mean[T, S <: Shape, A <: Shape](array: NDArray[T, S], axes: A): NDArray[T,

$\operatorname{Reduce}[\mathrm{S}, \mathrm{A}]]=$ ???

We implement reductions along a given list of indices with logic similar to two nested loops. The outer loop, Loop, traverses the shape and counts the current index. The inner loops are implemented using Contains and Remove, standard operations on HLists (omitted). When Loop reaches the end of the list, if there are still axes to remove, these are out of bounds for the initial shape: the match type intentionally gets stuck in such cases.

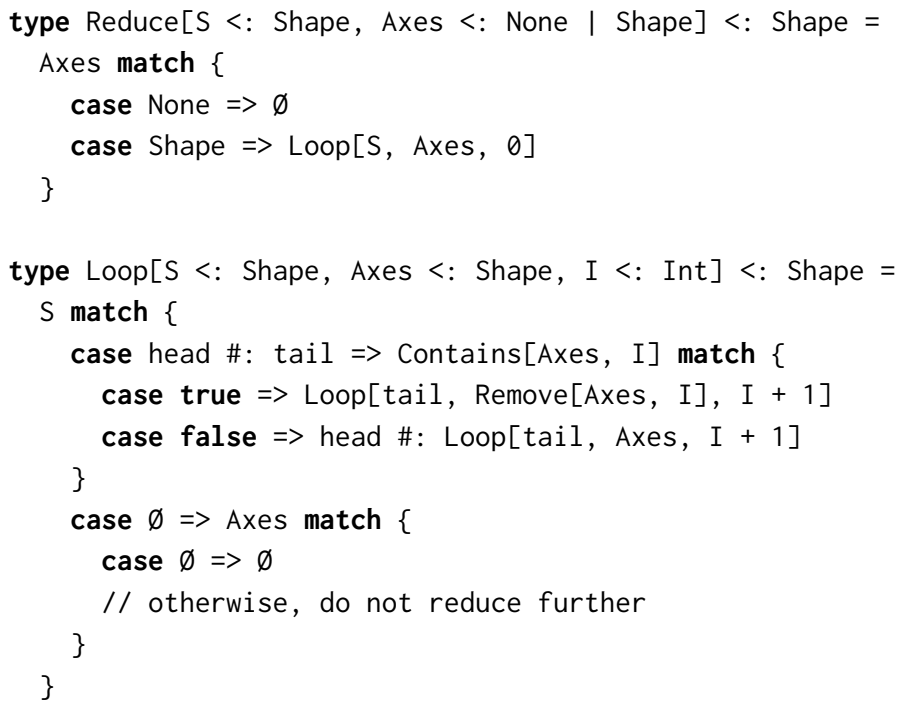




\subsection{Shape Safety}

Having defined random_normal, reshape and mean, we can rewrite our original Python example in Scala:

val img_batch $=$ random_normal $(\#:(25, \#:(256, \#:(256, \#:(3, \emptyset)))))$

val avg_colors $=$ mean (img_batch, \#: $(0, \#:(1, \#:(2, \varnothing))))$

val avg_color_square $=$ reshape $($ avg_colors, $\#:(5, \#:(5, \emptyset)))$

As expected, the call to mean returns a tensor of shape $3 \#$ : $\varnothing$. Therefore, the call to reshape does not type-check since the input shape has 3 elements instead of 25. If we fix the off by one error to reduce along the correct indices, $1 \#: 2 \#: 3 \#: \varnothing$, the call to reshape type-checks and avg_color has shape $25 \#$ : $\varnothing$, as expected.

\section{RELATED WORK}

In this section, we provide a review of existing work and relate match types to dependently typed calculi with subtyping, intensional type analysis, type families and roles in Haskell, and conditional types in TypeScript.

\subsection{Dependently Typed Calculi with Subtyping}

There is a vast amount of literature on type systems combining subtyping with dependent types, justifying a full survey to relate it appropriately. Instead, we offer a condensed summary of our reading journey and explain what led us to decide on using System $\mathrm{F}_{<:}$as a foundation for our formalization.

Dependently typed calculi typically use the same language to describe terms and types. This unification is also commonly used in the presence of subtyping [Hutchins 2010; Yang and Oliveira 2017; Zwanenburg 1999]. For systems with a complete term/type symmetry, this is a natural design, as it is concise and simplifies the meta-theory. Unfortunately, the lack of distinction between term and type level renders these systems impractical for our purpose, given that our research takes place in the context of an existing language with a clear term/type separation.

Singleton types provide an interesting middle ground between unified and separate syntax and have also been studied in conjunction with dependent types and subtyping [Aspinall 1995; Courant 2003; Stone and Harper 2000]. Singleton types give a mechanism to refer to terms in types, usually by means of a set-like syntax. This mechanism is appealing because it allows type system designers to cherry-pick the term constructs that should be allowed in types. When multiple constructs are shared between terms and types, singleton types provide a clear economy of concepts. In our study of match types, a minimal use of singleton types would result in sharing a single constructor between the term and the type languages: the constructor for matches. It is unclear if the benefits in doing so would outweigh the additional complexity.

Dependent Object Types (DOT) are, to this day, the most significant effort in formalizing Scala's type system [Amin et al. 2016]. DOT does not directly support any form of type-level computation. We considered using DOT as a starting point for our work, however, despite the recent effort to simplify DOT's soundness proof [Giarrusso et al. 2020; Rapoport et al. 2017], extending DOT remains too big of a challenge to concisely describe language extensions.

After several attempts at formalizing match types within existing systems, we decided to pursue a simpler route of adding new constructs to a system without dependent types. After all, the primary purpose of System FM is to serve as a medium to concisely explain our type-checking algorithm for match types. For this reason, we built our work on top of System $\mathrm{F}_{<:}$, which we believe should be the simplest, most familiar calculus among the systems cited in this section. 


\subsection{Intensional Type Analysis}

In their work on intensional type analysis [Harper and Morrisett 1995], Harper and Morrisett introduce the $\lambda_{i}^{M L}$ calculus that supports structural analysis of types. In $\lambda_{i}^{M L}$, types are represented as expressions that can be inspected by case analysis using a "typecase" construct, available both at the term and at the type level. Match types can be seen as an extension of intensional type analysis to work with object-oriented class hierarchies and subtyping. Whereas patterns in $\lambda_{i}^{M L}$ are limited to a fixed set of disjoint types, match types need to deal with open class hierarchies, of which not all members are known at compile-time. This means pattern types can overlap, and we need to perform an analysis of disjointness between the scrutinee type and each pattern type. Disjointness allows for sound reduction in the presence of overlapping patterns and abstract scrutinee types, while retaining the natural sequential evaluation order of pattern matching.

\subsection{Type Families in Haskell}

Haskell's type families allow programmers to define type-level functions using pattern matching [Chakravarty et al. 2005; Schrijvers et al. 2008]. By default, type families are open, which means that a definition can spread across multiple files and compilations. This flexibility induces a substantial restriction on type family definitions: patterns must not overlap. One benefit of this restriction is that it prevents any ambiguity in the reduction of type families (patterns are pairwise disjoint), which is required given the distributed nature of definitions. Open type families are well-suited to be used in conjunction with type classes, since both constructs have open-ended definitions with non-overlapping constraints.

Closed type families (CTFs), as introduced by Eisenberg et al. [2014], allow for overlapping cases in type family definitions. Unlike the open variant, CTF reduction is performed sequentially, based on unification and apartness checks. In this regard, CTFs are closely related to match types. In fact, if we replace unification checks with subtyping and apartness checks with disjointness, their reduction algorithm is practically identical to ours, from a high-level perspective.

By default, Haskell checks for termination of recursive type families, but this check can be disabled to increase type families' expressiveness. Although the formalization presented in [Eisenberg et al. 2014] does not cover non-terminating families, the paper discusses a soundness problem caused by non-termination. The problem only occurs in the presence of repeated type bindings in patterns. In Scala, match types (and pattern matching in general) do not allow repeated bindings and are therefore not affected by this problem.

\subsection{Roles in Haskell}

Haskell's roles were introduced by Weirich et al. [2011] to fix a long-standing unsoundness caused by the interaction of open type families and the newtype construct. We understand roles as type annotations which specify whether a given type can safely be nominally compared to other types, or if representational equality (RE) should be used instead. The word representation in RE refers to the runtime representation of a type. In particular, RE dealiases newtype constructs.

In their introductory example, Weirich et al. show how type family reduction can lead to unsoundness in the absence of role annotations. Their presentation also includes a hypothetical translation of their example to Standard ML, which translates directly to Scala as follows:

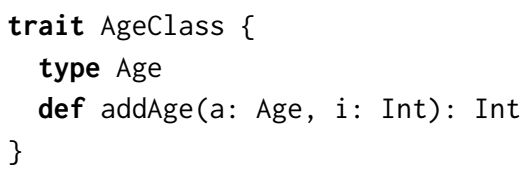




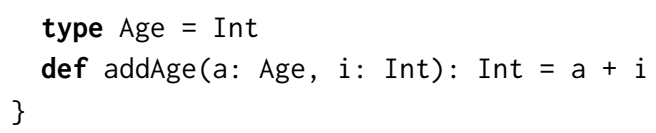

In this example, type Age is abstract in AgeClass and concrete in AgeObject. In the pre-role Haskell equivalent of this example, the unsoundness comes when the above definitions are combined with a type family that discriminates Age and Int. Such type family would reduce differently in AgeClass, where the types are different, and in AgeObject, where those two types are synonyms, which can easily be exploited to obtain a runtime error.

Luckily, our design of match types is not affected by this issue. The reason comes from the use of subtyping (and disjointness), which shields our implementation from incorrectly discriminating Age from Int when Age is abstract. Consider the following match type definition (directly translated from the Haskell example):

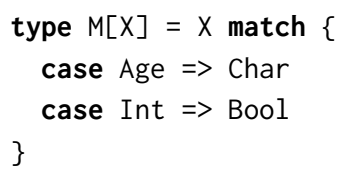

Our algorithm would not reduce M[Int ] to Bool in AgeClass as this reduction would require evidence that Age and Int are disjoint, which cannot be constructed when Age is an unbounded abstract type.

\subsection{Conditional Types in TypeScript}

TypeScript's conditional types, briefly mentioned in the introduction, are a type-level ternary operator based on subtyping. Conditional types can be nested into a sequence of patterns that evaluate in order, making them similar to match types.

The TypeScript language specification briefly describes the algorithm used to reduce conditional types in the presence of type variables [TypeScript development team 2020]. Given a type $S$ extends $T$ ? Tt : Tf, the TypeScript compiler first replaces all the type parameters in $S$ and $T$ by any (the top of TypeScript's subtyping lattice). If the resulting types (after substitution) are not subtypes, the overall condition is reduced to Tf. Unfortunately, this algorithm is both unsound and incomplete.

The unsoundness is caused by the incorrect widening of type parameters in contravariant position. Although TypeScript does not have syntax for variance annotations, function types are covariant in their return type and contravariant in their arguments. The conditional type unification algorithm wrongly approximates $X=>$ string to any $\Rightarrow>$ string and unifies the former with latter, which can lead to a runtime errors.

The incompleteness comes from the fact that type parameter approximation does not account for type parameter bounds. Consider the following example:

type $M<X>=X$ extends string ? $A: B$

function $f<X$ extends string $>$ : $M<X>=$ new $A$

Here, TypeScript's reduction algorithm fails to recognize that new A can be typed as $M<X>$, even though X is clearly a subtype of string in f's body.

Although the situation is concerning, it might not be as bad as it seems given that soundness is a non-goal of TypeScript's type system [Bierman et al. 2014]. Nevertheless, we believe that the results of this paper are directly applicable to conditional types and could be used to improve TypeScript's type checker. 


\section{CONCLUSION}

In this paper, we introduced match types, a lightweight mechanism for type-level programming that integrates seamlessly in subtyping-based programming languages. We formalized match types in System FM, a calculus based on System $\mathrm{F}_{<:}$, and proved it sound. Furthermore, we implemented match types in the Scala 3 compiler, making them readily available to a large audience of programmers. A key insight for sound match types is the notion of disjointness, which complements subtyping in the match type reduction algorithm. In the future, we plan to investigate inference of match types to avoid code duplication in programs that operate both at the term and the type level.

\section{REFERENCES}

Martín Abadi, Luca Cardelli, Benjamin Pierce, and Gordon Plotkin. 1991. Dynamic Typing in a Statically Typed Language. ACM Trans. Program. Lang. Syst. 13, 2 (April 1991), 237-268. https://doi.org/10.1145/103135.103138

Nada Amin, Samuel Grütter, Martin Odersky, Tiark Rompf, and Sandro Stucki. 2016. The Essence of Dependent Object Types. Springer International Publishing, Cham, 249-272. https://doi.org/10.1007/978-3-319-30936-1_14

David Aspinall. 1995. Subtyping with singleton types. In International Workshop on Computer Science Logic. Springer, Springer Berlin Heidelberg, Berlin, Heidelberg, 1-15. https://doi.org/10.1007/BFb0022243

Brian Aydemir, Arthur Charguéraud, Benjamin C. Pierce, Randy Pollack, and Stephanie Weirich. 2008. Engineering Formal Metatheory. In Proceedings of the 35th Annual ACM SIGPLAN-SIGACT Symposium on Principles of Programming Languages (San Francisco, California, USA) (POPL'08). Association for Computing Machinery, New York, NY, USA, 3-15. https://doi.org/10.1145/1328438.1328443

Paul Barham and Michael Isard. 2019. Machine Learning Systems Are Stuck in a Rut. In Proceedings of the Workshop on Hot Topics in Operating Systems (Bertinoro, Italy) (HotOS '19). ACM, New York, NY, USA, 177-183. https://doi.org/10.1145/ 3317550.3321441

Gavin Bierman, Martín Abadi, and Mads Torgersen. 2014. Understanding TypeScript. In Proceedings of the 28th European Conference on ECOOP 2014 - Object-Oriented Programming - Volume 8586. Springer-Verlag, Berlin, Heidelberg, 257-281. https://doi.org/10.1007/978-3-662-44202-9_11

Olivier Blanvillain, Jonathan Brachthäuser, Maxime Kjaer, and Martin Odersky. 2021a. Type-Level Programming with Match Types. (2021), 70. http://infoscience.epfl.ch/record/290019

Olivier Blanvillain, Jonathan Brachthäuser, Maxime Kjaer, and Martin Odersky. 2021b. Type-Level Programming with Match Types Artifact. https://doi.org/10.5281/zenodo.5568850

Olivier Blanvillain, Marios Iliofotou, Adelbert Chang, Gleb Kanterov, and other open-source contributors. 2016-2021c. Frameless. https://github.com/typelevel/frameless.

Luca Cardelli, Simone Martini, John C Mitchell, and Andre Scedrov. 1994. An extension of system F with subtyping. Information and computation 109, 1-2 (1994), 4-56. https://doi.org/10.1006/inco.1994.1013

Manuel M. T. Chakravarty, Gabriele Keller, and Simon Peyton Jones. 2005. Associated Type Synonyms. SIGPLAN Not. 40, 9 (Sept. 2005), 241-253. https://doi.org/10.1145/1090189.1086397

Tongfei Chen. 2017. Typesafe Abstractions for Tensor Operations (Short Paper). In Proceedings of the 8th ACM SIGPLAN International Symposium on Scala (Vancouver, BC, Canada) (SCALA 2017). Association for Computing Machinery, New York, NY, USA, 45-50. https://doi.org/10.1145/3136000.3136001

Judicaël Courant. 2003. Strong normalization with singleton types. Electronic Notes in Theoretical Computer Science 70, 1 (2003), 53-71. https://doi.org/10.1016/S1571-0661(04)80490-0

Richard A Eisenberg. 2016. Dependent types in Haskell: Theory and practice. University of Pennsylvania.

Richard A. Eisenberg, Dimitrios Vytiniotis, Simon Peyton Jones, and Stephanie Weirich. 2014. Closed Type Families with Overlapping Equations. In Proceedings of the 41st ACM SIGPLAN-SIGACT Symposium on Principles of Programming Languages (San Diego, California, USA) (POPL'14). Association for Computing Machinery, New York, NY, USA, 671-683. https://doi.org/10.1145/2535838.2535856

Burak Emir, Martin Odersky, and John Williams. 2007. Matching Objects with Patterns. In Proceedings of the 21st European Conference on Object-Oriented Programming (Berlin, Germany) (ECOOP'07). Springer-Verlag, Berlin, Heidelberg, 273-298. https://doi.org/10.1007/978-3-540-73589-2_14

Paolo G. Giarrusso, Léo Stefanesco, Amin Timany, Lars Birkedal, and Robbert Krebbers. 2020. Scala Step-by-Step: Soundness for DOT with Step-Indexed Logical Relations in Iris. Proc. ACM Program. Lang. 4, ICFP, Article 114 (2020), 29 pages. https://doi.org/10.1145/3408996

Robert Harper and Greg Morrisett. 1995. Compiling Polymorphism Using Intensional Type Analysis. In Proceedings of the 22nd ACM SIGPLAN-SIGACT Symposium on Principle s of Programming Languages (San Francisco, California, USA) (POPL '95). Association for Computing Machinery, New York, NY, USA, 130-141. https://doi.org/10.1145/199448.199475 
Charles R. Harris, K. Jarrod Millman, Stéfan J. van der Walt, Ralf Gommers, Pauli Virtanen, David Cournapeau, Eric Wieser, Julian Taylor, Sebastian Berg, Nathaniel J. Smith, Robert Kern, Matti Picus, Stephan Hoyer, Marten H. van Kerkwijk, Matthew Brett, Allan Haldane, Jaime Fernández del Río, Mark Wiebe, Pearu Peterson, Pierre Gérard-Marchant, Kevin Sheppard, Tyler Reddy, Warren Weckesser, Hameer Abbasi, Christoph Gohlke, and Travis E. Oliphant. 2020. Array programming with NumPy. Nature 585, 7825 (Sept. 2020), 357-362. https://doi.org/10.1038/s41586-020-2649-2

Austin Huang, Sam Stites, and Torsten Scholak. 2017-2021. HaskTorch. https://github.com/hasktorch/hasktorch.

DeLesley S. Hutchins. 2010. Pure Subtype Systems. In Proceedings of the 37th Annual ACM SIGPLAN-SIGACT Symposium on Principles of Programming Languages (Madrid, Spain) (POPL'10). Association for Computing Machinery, New York, NY, USA, 287-298. https://doi.org/10.1145/1706299.1706334

Oleg Kiselyov, Ralf Lämmel, and Keean Schupke. 2004. Strongly Typed Heterogeneous Collections. In Proceedings of the 2004 ACM SIGPLAN Workshop on Haskell (Snowbird, Utah, USA) (Haskell '04). Association for Computing Machinery, New York, NY, USA, 96-107. https://doi.org/10.1145/1017472.1017488

George Leontiev, Eugene Burmako, Jason Zaugg, Adriaan Moors, Paul Phillips, Oron Port, and Miles Sabin. 2014. SIP-23 Literal-Based Singleton Types. Scala Center. https://docs.scala-lang.org/sips/42.type.html

Fengyun Liu. 2016. A Generic Algorithm for Checking Exhaustivity of Pattern Matching (Short Paper). In Proceedings of the 2016 7th ACM SIGPLAN Symposium on Scala (Amsterdam, Netherlands) (SCALA 2016). Association for Computing Machinery, New York, NY, USA, 61-64. https://doi.org/10.1145/2998392.2998401

Abel Nieto, Yaoyu Zhao, Ondřej Lhoták, Angela Chang, and Justin Pu. 2020. Scala with Explicit Nulls. In 34th European Conference on Object-Oriented Programming (ECOOP 2020) (Leibniz International Proceedings in Informatics (LIPIcs), Vol. 166), Robert Hirschfeld and Tobias Pape (Eds.). Schloss Dagstuhl-Leibniz-Zentrum für Informatik, Dagstuhl, Germany, 25:1-25:26. https://doi.org/10.4230/LIPIcs.ECOOP.2020.25

Benjamin C. Pierce. 2002. Types and programming languages. MIT press.

Michael Pilquist and Scodec open-source contributors. 2013-2021. Scodec. https://github.com/scodec/scodec.

Marianna Rapoport, Ifaz Kabir, Paul He, and Ondvrej Lhoták. 2017. A Simple Soundness Proof for Dependent Object Types. Proc. ACM Program. Lang. 1, OOPSLA, Article 46 (Oct. 2017), 27 pages. https://doi.org/10.1145/3133870

Alexander Rush. 2019. Tensor Considered Harmful. Harvard NLP. https://nlp.seas.harvard.edu/NamedTensor

Miles Sabin and Shapeless open-source contributors. 2011-2021. Shapeless. https://github.com/milessabin/shapeless.

Michel Schinz. 2005. Compiling Scala for the fava virtual machine. Ph.D. Dissertation. EPFL, Lausanne. https://doi.org/10. 5075/epfl-thesis-3302

Tom Schrijvers, Simon Peyton Jones, Manuel Chakravarty, and Martin Sulzmann. 2008. Type Checking with Open Type Functions. In Proceedings of the 13th ACM SIGPLAN International Conference on Functional Programming (Victoria, BC, Canada) (ICFP'08). Association for Computing Machinery, New York, NY, USA, 51-62. https://doi.org/10.1145/1411204. 1411215

Vilhelm Sjoberg. 2015. A Dependently Typed Language with Nontermination. Ph.D. Dissertation. University of Pennsylvania.

Christopher A. Stone and Robert Harper. 2000. Deciding Type Equivalence in a Language with Singleton Kinds. In Proceedings of the 27th ACM SIGPLAN-SIGACT Symposium on Principles of Programming Languages (Boston, MA, USA) (POPL'00). Association for Computing Machinery, New York, NY, USA, 214-227. https://doi.org/10.1145/325694.325724

TypeScript development team. 2020. The TypeScript Handbook. Microsoft Corporation. https://www.typescriptlang.org/

Stephanie Weirich, Dimitrios Vytiniotis, Simon Peyton Jones, and Steve Zdancewic. 2011. Generative Type Abstraction and Type-Level Computation. In Proceedings of the 38th Annual ACM SIGPLAN-SIGACT Symposium on Principles of Programming Languages (Austin, Texas, USA) (POPL'11). Association for Computing Machinery, New York, NY, USA, 227-240. https://doi.org/10.1145/1926385.1926411

Yanpeng Yang and Bruno C. d. S. Oliveira. 2017. Unifying Typing and Subtyping. Proc. ACM Program. Lang. 1, OOPSLA, Article 47 (2017), 26 pages. https://doi.org/10.1145/3133871

Jan Zwanenburg. 1999. Pure type systems with subtyping. In International Conference on Typed Lambda Calculi and Applications. Springer Berlin Heidelberg, Berlin, Heidelberg, 381-396. https://doi.org/10.1007/3-540-48959-2_27

Proc. ACM Program. Lang., Vol. 6, No. POPL, Article 37. Publication date: January 2022. 